\title{
The effective temperature scale of giant stars (F0-K5)
}

\section{Empirical calibration of $T_{\text {eff }}$ versus colours and $[\mathrm{Fe} / \mathrm{H}]$}

\author{
A. Alonso, S. Arribas, and C. Martínez-Roger \\ Instituto de Astrofísica de Canarias, E-38200 La Laguna, Tenerife, Spain \\ e-mail: aas@ll.iac.es, sam@ll.iac.es and cmr@ll.iac.es
}

Received March 19; accepted July 23, 1999

\begin{abstract}
We present calibrations of the effective temperatures of giant stars versus $[\mathrm{Fe} / \mathrm{H}]$ and colours $(U-V)$, $(B-V),(R-I),(V-R),(V-I),(V-K),(J-H)$, $(J-K),(I-K),\left(V-L^{\prime}\right),(b-y)$ and $(u-b)$. These calibrations are based on a large sample of field and globular cluster stars which roughly cover spectral types from F0 to K5. Their effective temperatures, scaled to direct $T_{\text {eff }}$ determinations via reliable angular diameter measurements, were derived by applying the infrared flux method. The empirical relations have been fitted to polynomials of the form $\theta_{\text {eff }}=P($ colour, $[\mathrm{Fe} / \mathrm{H}])$ by using the least squares method. The precision of the fits ranges from $40 \mathrm{~K}$ for $(V-K)$ to $170 \mathrm{~K}$ for $(J-H)$. We tabulate intrinsic colours of giant stars in the ranges: $3500 \mathrm{~K} \leq T_{\text {eff }} \leq 8000 \mathrm{~K}$; $-3.0 \leq[\mathrm{Fe} / \mathrm{H}] \leq+0.5$. We also present the calibration of $\mathrm{BC}(\mathrm{V})$ as a function of $\log \left(T_{\text {eff }}\right)$ and metallicity. Finally, we compare the resulting scale of temperatures with previous works.
\end{abstract}

Key words: stars: fundamental parameters - stars: Population II — stars: giants — stars: atmospheres stars: general

\section{Introduction}

The calibration of the scale of effective temperatures of stars (i.e. relations which link $T_{\text {eff }},[\mathrm{Fe} / \mathrm{H}]$ and explicitly or implicitly $\log (g)$ with observable features such as photometric colours and indices or spectral features) is a necessary tool in many astrophysical fields for interpreting observations or relating them with theory. Affected topics range from stellar physics to cosmology. Four general areas can be readily mentioned:

The interpretation of the observed HR diagram (e.g., $V: B-V)$ in terms of theoretical isochrones $\left(L: T_{\text {eff }}\right)$;

Send offprint requests to: A. Alonso
In this case, the morphology of red giant branch and lower main sequence is appreciably altered by changes of $\sim 100-200 \mathrm{~K}$ in the effective temperature-colour calibration adopted (e.g. Bell 1992; Cassisi et al. 1999).

Another important area affected by $T_{\text {eff }}$ calibrations is the determination of chemical abundances from spectroscopic analysis. As a rather conspicuous example of "cosmological" consequences, an erroneous temperature scale for dwarfs could lead to incorrect conclusions regarding the primordial lithium abundance (e.g. Spite et al. 1998; Bonifacio \& Molaro 1997 versus Ryan et al. 1996). Regarding giants, the influence of temperature on the strength of molecular bands in cool stars is well known and it is equally important for the determination of neutral element abundances; temperature indicators independent of metallicity and only weakly influenced by interstellar reddening are therefore needed (e.g. Sneden et al. 1992).

The analysis of the physics of stellar atmosphere models also requires the use of empirical temperatures to avoid the risk of a vicious circle (e.g., Bell \& Gustaffson 1989).

Finally, in the synthesis of stellar populations, the use of colours and spectral atlases requires an accurate determination of atmospheric parameters and of the effective temperature in particular (e.g. Vazdekis et al. 1996). As a consequence the calibration of $T_{\text {eff }}$ with colour and metallicity is also relevant in these types of (extra)galactic studies.

In conclusion, analyses in several astrophysical fields require now stringent internal accuracies of the effective temperatures, typically of the order of $1 \%$.

The calibration of the temperature scale of giant stars of Population I with semi-empirical approaches has been addressed in several works (e.g. Ridgway et al. 1980), however the extension to Population II has been only fully and homogeneously accomplished by means of theoretical methods (e.g. Bessell et al. 1998). The calibrations we present here cover temperature and metallicity in the ranges: $3500 \mathrm{~K} \leq T_{\text {eff }} \leq 8000 \mathrm{~K} ;-3.0 \leq[\mathrm{Fe} / \mathrm{H}] \leq$ 
+0.5 . Therefore, the present study extends previous semiempirical works towards the metal-poor domain providing calibrations with a smaller dependence on atmosphere models.

This work is part of a long term programme aimed at a complete and uniform revision of the $T_{\text {eff }}$ scale of the different regions of the HR diagram. It is based on temperatures derived with the InfraRed Flux Method (IRFM, Blackwell et al. 1990), scaled to direct $T_{\text {eff }}$, and on good quality photoelectric photometry. The subprogramme concerning giant stars in which the present work is included has been extensively explained in (Alonso et al. 1999; Paper II).

\section{The multiparametric calibrations}

The effective temperatures used for the present set of calibrations were uniformly obtained by means of the IRFM as described in Paper II. The sources of photometry are documented in Alonso et al. (1998; Paper I), critical attention has been paid to the homogenization of data extracted from different catalogues, and to the correction of interstellar reddening. It is worth noticing that the composition of the sample reflects a good balance between field and globular cluster giants.

In Table 1, we present the effective temperature scale of giant stars (class III) from early $\mathrm{F}$ to late $\mathrm{K}$, which results from averaging temperatures of the stars of the sample classified in each spectral type. The utility of this kind of table is limited since on the one hand it requires the knowledge of the spectral type of the problem star, which is not always available; on the other, the relation between temperature and spectral type depends also on metallicity, and only a restricted number of metal-poor giants have an accurate spectral type classification (the use of published spectral classifications is too coarse as proven by the size of errorbars in Table 1). For this reason we provide in sections below more useful calibrations of the temperature scale against direct photometric observables and $[\mathrm{Fe} / \mathrm{H}]$.

From a practical standpoint we have fitted the data to polynomials of the form $\theta_{\text {eff }}=P($ colour, $[\mathrm{Fe} / \mathrm{H}])$, where $\theta_{\text {eff }}=5040 / T_{\text {eff }}$. In a preliminary step, a group of stars which departed from the mean tendency in several colourcolour diagrams were discarded from the sample used in the calibration. The least squares method was then iteratively applied discarding in successive steps stars which departed from the fit more than $2.5 \sigma$ taking care that residuals were more or less normally distributed. Typically, four to seven iterations were enough depending on the colour used in the calibration. This approach has been previously applied with good results for the calibration of main sequence temperatures (Alonso et al. 1996; Paper III). In Table 2, we summarize the coefficients of the fits. In Table 3 we show the colour and metallicity ranges of applicability of each of the fits. Finally, we show in Table 4 the stars discarded in any of the fits.

\section{1. $T_{\mathrm{eff}}:[\mathrm{Fe} / \mathrm{H}]:(U-V)$ and $:(B-V)$}

Johnson's $U B V$ photometry is practically extended to all stars contained in astronomical catalogues of interest, for this reason the calibration of $(B-V)$ and $(U-V)$ as temperature indicators is useful in many studies. We have adopted $(U-V)$ instead of $(U-B)$, which is more commonly tabulated, because it is a better indicator of temperature and has a similar behaviour with metallicity.

The fits obtained for $T_{\text {eff }}:[\mathrm{Fe} / \mathrm{H}]:(U-V)$ are shown in Table 2 (Eqs. (1) and (2)); the corresponding ranges of application are shown in Table 3 .

In the range of colour $1.2 \leq(U-V) \leq 1.5$ a linear interpolation of Eqs. (1) and (2) provides a good fit of the data ensuring continuity.

The fits obtained for $T_{\text {eff }}:[\mathrm{Fe} / \mathrm{H}]:(B-V)$ are shown in Table 2 (Eqs. (3) and (4)); the corresponding ranges of application are shown in Table 3.

In the range of colour $0.7 \leq(B-V) \leq 0.8$ a linear interpolation of Eqs. (3) and (4) provides a good fit and ensures continuity.

A caveat has to be pointed out about these calibrations in the range of low temperatures: At $(B-V) \approx 1.55$ and $(U-V) \approx 3.55$ temperature seems to drop abruptly $\sim 250 \mathrm{~K}$. This effect could be real, probably related to the variation of surface gravity in this range. The ultimate reason being a variation of the flux balance in the $\mathrm{UV} /$ visible region linked to changes in the opacity sources with gravity. In this respect, a turn-over is observed in the colour:colour diagrams $(V-K):(B-V)$ and $(V-K):(U-V)$, which would imply constant colour with decreasing temperature (see Figs. 8 and 9 in Paper I). However, another possible explanation could be in the shortcomings of models below $4000 \mathrm{~K}$. Unfortunately, temperatures derived by means of the IRFM in this range are affected by large errors which make difficult to ascertain if the effect is spurious or not. In any case, the polynomial fits used here are obviously unable to follow the described feature. Therefore, under $4000 \mathrm{~K}$ this point should be taken into account when applying the above calibrations.

We display in Figs. 1 and 2 the residuals of the fits. The observed dispersion is compatible with typical errors on $T_{\text {eff }},[\mathrm{Fe} / \mathrm{H}],(U-V)$ and $(B-V)$.

The mean variation $\Delta T_{\text {eff }} / \Delta(U-V)$ amounts approximately to $18 \mathrm{~K}$ per $0.01 \mathrm{mag}$ for $(U-V)<1.0$ and $6 \mathrm{~K}$ per $0.01 \mathrm{mag}$ for $(U-V)>1.0$. At constant $(U-V)$ temperature monotonically decreases with decreasing $[\mathrm{Fe} / \mathrm{H}]$. The gradient $\Delta T_{\text {eff }} /[\mathrm{Fe} / \mathrm{H}]$ depends slightly on colour and diminishes with decreasing $[\mathrm{Fe} / \mathrm{H}]$, as expected from atmospheres theory. The value of saturation is out of the range of the present calibration, although extrapolation 
Table 1. Effective temperature scale for class III stars. Data between parentheses mean that the number of stars considered in the average was too small as to provide a significant standard deviation

\begin{tabular}{c|c|c|c}
\hline \hline & \multicolumn{3}{|c}{$T_{\text {eff }}(\mathrm{K})$} \\
\hline Sp. type & $0.5 \gtrsim[\mathrm{Fe} / \mathrm{H}] \gtrsim-1.0$ & $-1.0 \gtrsim[\mathrm{Fe} / \mathrm{H}] \gtrsim-2.0$ & {$[\mathrm{Fe} / \mathrm{H}] \lesssim-2.0$} \\
\hline F0III & $7046 \pm 300$ & - & - \\
F2III & $6804 \pm 300$ & - & - \\
F5III & $6255 \pm 55$ & - & - \\
F6III & $6190 \pm 115$ & - & $4920 \pm 100$ \\
F8III & $5805 \pm 225$ & - & $4875 \pm 235$ \\
G0III & - & $5306 \pm 265$ & $4753 \pm 450$ \\
G2III & - & - & - \\
G4III & $5050 \pm 155$ & - & - \\
G5III & $5040 \pm 100$ & - & - \\
G6III & $4920 \pm 155$ & - & - \\
G7III & $4860 \pm 130$ & - & - \\
G8III & $4725 \pm 230$ & - & - \\
G9III & $4660 \pm 180$ & - & - \\
K0III & $4580 \pm 245$ & - & - \\
K1III & $4455 \pm 190$ & - & - \\
K2III & $4285 \pm 160$ & - & - \\
K3III & $4195 \pm 320$ & - & - \\
K4III & $3950 \pm 115$ & K5III &
\end{tabular}

Table 2. Coefficients for the fits of the form $\theta_{\mathrm{eff}}=a_{0}+a_{1} X+a_{2} X^{2}-a_{3} X[\mathrm{Fe} / \mathrm{H}]+a_{4}[\mathrm{Fe} / \mathrm{H}]+a_{5}[\mathrm{Fe} / \mathrm{H}]^{2}$, where $X$ stands for the colour (Col. 2). The corresponding standard deviations $\sigma\left(\theta_{\text {eff }}\right)$ and $\sigma\left(T_{\text {eff }}\right)$, together with the number of stars considered, are also shown. Column 1 contains the equation number assigned to each fit in the text

\begin{tabular}{|c|c|c|c|c|c|c|c|c|c|c|}
\hline Eq. \# & Colour & $a_{0}$ & $a_{1}$ & $a_{2}$ & $a_{3}$ & $a_{4}$ & $a_{5}$ & $\sigma\left(\theta_{\text {eff }}\right)$ & $\sigma\left(T_{\text {eff }}\right)(\mathrm{K})$ & N. of stars \\
\hline 1 & $(U-V)$ & 0.6388 & 0.4065 & -0.1117 & $-2.308 \mathrm{e}-3$ & $-7.783 \mathrm{e}-2$ & $-1.200 \mathrm{e}-2$ & 0.023 & 164 & 127 \\
\hline 2 & $(U-V)$ & 0.8323 & $9.374 \mathrm{e}-2$ & $1.184 \mathrm{e}-2$ & $2.351 \mathrm{e}-2$ & -0.1392 & $-1.944 \mathrm{e}-2$ & 0.020 & 80 & 283 \\
\hline 3 & $(B-V)$ & 0.5716 & 0.5404 & $-6.126 \mathrm{e}-2$ & $-4.862 \mathrm{e}-2$ & $-1.777 \mathrm{e}-2$ & $-7.969 \mathrm{e}-3$ & 0.020 & 167 & 122 \\
\hline 4 & $(B-V)$ & 0.6177 & 0.4354 & $-4.025 \mathrm{e}-3$ & $5.204 \mathrm{e}-2$ & -0.1127 & $-1.385 \mathrm{e}-2$ & 0.024 & 96 & 416 \\
\hline 5 & $(V-R)$ & 0.4972 & 0.8841 & -0.1904 & $-1.197 \mathrm{e}-2$ & $-1.025 \mathrm{e}-2$ & $-5.500 \mathrm{e}-3$ & 0.021 & 150 & 248 \\
\hline 6 & $(V-I)^{*}$ & $\theta_{\mathrm{eff}}=$ & $=0.5379+$ & $+0.3981(V$ & $-I)+4.43$ & $2 \mathrm{e}-2(V-I)^{2}$ & $-2.693 \mathrm{e}-2(V-I)^{3}$ & 0.017 & 125 & 214 \\
\hline 7 & $(R-I)$ & 0.4974 & 1.345 & -0.5008 & $-8.134 \mathrm{e}-2$ & $3.705 \mathrm{e}-2$ & $-6.184 \mathrm{e}-3$ & 0.022 & 150 & 217 \\
\hline 8 & $(V-K)$ & 0.5558 & 0.2105 & $1.981 \mathrm{e}-3$ & $-9.965 \mathrm{e}-3$ & $1.325 \mathrm{e}-2$ & $-2.726 \mathrm{e}-3$ & 0.005 & 40 & 256 \\
\hline 9 & $(V-K)$ & 0.3770 & 0.3660 & $-3.170 \mathrm{e}-2$ & $-3.074 \mathrm{e}-3$ & $-2.765 \mathrm{e}-3$ & $-2.973 \mathrm{e}-3$ & 0.005 & 25 & 412 \\
\hline 10 & $(J-H)$ & 0.5977 & 1.015 & $-1.020 \mathrm{e}-1$ & $-1.029 \mathrm{e}-2$ & $3.006 \mathrm{e}-2$ & $1.013 \mathrm{e}-2$ & 0.023 & 170 & 505 \\
\hline 11 & $(J-K)$ & 0.5816 & 0.9134 & -0.1443 & 0.0000 & 0.0000 & 0.0000 & 0.020 & 125 & 511 \\
\hline 12 & $\left(V-L^{\prime}\right)^{*}$ & $\theta_{\text {eff }}=0$ & $0.5641+$ & $0.1882(V-$ & $\left.L^{\prime}\right)+1.89($ & e $-2\left(V-L^{\prime}\right)^{2}$ & $-4.651 \mathrm{e}-3\left(V-L^{\prime}\right)^{3}$ & 0.009 & 65 & 122 \\
\hline 13 & $(I-K)_{J}$ & 0.5859 & 0.4846 & $-2.457 \mathrm{e}-2$ & 0.0000 & 0.0000 & 0.0000 & 0.018 & 130 & 213 \\
\hline 14 & $(b-y)$ & 0.5815 & 0.7263 & $6.856 \mathrm{e}-2$ & $-6.832 \mathrm{e}-2$ & $-1.062 \mathrm{e}-2$ & $-1.079 \mathrm{e}-2$ & 0.013 & 110 & 118 \\
\hline 15 & $(b-y)$ & 0.4399 & 1.209 & -0.3541 & $8.443 \mathrm{e}-2$ & -0.1063 & $-1.686 \mathrm{e}-2$ & 0.018 & 70 & 169 \\
\hline 16 & $(u-b)$ & 0.5883 & 0.2008 & $-5.931 \mathrm{e}-3$ & $5.319 \mathrm{e}-3$ & $-1.000 \mathrm{e}-1$ & $-1.542 \mathrm{e}-2$ & 0.021 & 110 & 181 \\
\hline
\end{tabular}

* The functional expression of the fit for this colour is explicitly shown, since it differs from the general expression adopted.

provides $[\mathrm{Fe} / \mathrm{H}] \approx-3.5$. When using this calibration an error of 0.05 mag on measured $(U-V)$ implies mean errors of $1.5-0.7 \%$ in temperature. Equivalently, an error of 0.5 dex in $[\mathrm{Fe} / \mathrm{H}]$ implies mean errors of $3.5-2.3 \%$.

The mean variation $\Delta T_{\text {eff }} / \Delta(B-V)$ amounts approximately to $42 \mathrm{~K}$ per $0.01 \mathrm{mag}$ for $(B-V)<0.8$ and $15 \mathrm{~K}$ per $0.01 \mathrm{mag}$ for $(B-V)>0.8$. At constant $(B-V)$, temperature monotonically decreases with decreasing $[\mathrm{Fe} / \mathrm{H}]$. The gradient $\Delta T_{\text {eff }} / \Delta[\mathrm{Fe} / \mathrm{H}]$ depends on colour and tends to zero as $[\mathrm{Fe} / \mathrm{H}]$ decreases (saturation occurring at
$[\mathrm{Fe} / \mathrm{H}] \approx-3)$. When using this calibration an error of $0.03 \mathrm{mag}$ on $(B-V)$ implies mean errors of $1.2-2.0 \%$ in temperature. Equivalently, an error of 0.5 dex in $[\mathrm{Fe} / \mathrm{H}]$ implies mean errors ranging $1.1-1.9 \%$.

In Fig. 3, we show the comparison of calibrations (1), (2), (3) and (4) with several representative calibrations published previously.

We have included in our analysis the scale of Johnson (1996; J66) because, from a historic point of view, it is the first comprehensive calibration of the temperature scale, 


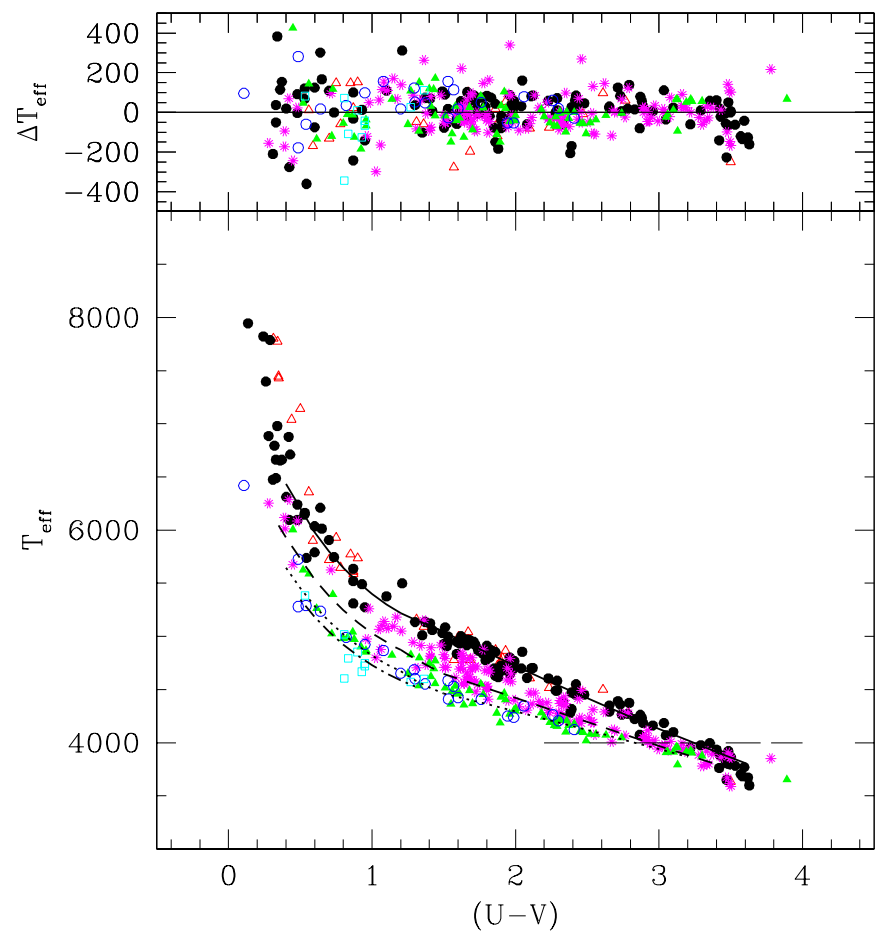

Fig. 1. $T_{\text {eff }}$ against $(U-V)$. The lines display the fit corresponding to Eqs. (1) and (2) for $[\mathrm{Fe} / \mathrm{H}]=0$ (solid line), $[\mathrm{Fe} / \mathrm{H}]=-1$ (dashed line), $[\mathrm{Fe} / \mathrm{H}]=-2$ (dotted line), $[\mathrm{Fe} / \mathrm{H}]=-3$ (dashed-dotted line). Symbols stand for the following metallicity groups: Open triangles $[\mathrm{Fe} / \mathrm{H}]>0$, filled circles $0 \geq[\mathrm{Fe} / \mathrm{H}]>-0.25$, asterisks $-0.25 \geq[\mathrm{Fe} / \mathrm{H}]>-1$, filled triangles $-1 \geq[\mathrm{Fe} / \mathrm{H}]>-2$, open circles $-2 \geq[\mathrm{Fe} / \mathrm{H}]>-2.5$, squares $-2.5 \geq[\mathrm{Fe} / \mathrm{H}]$. The horizontal long-dashed line delineates the region $T_{\text {eff }} \leq 4000 \mathrm{~K}$ where temperatures derived by means of the IRFM have lower accuracy. The top panel of the figure shows the residuals of the fit

although his results have been superseded by more recent works. It is remarkable the good agreement found both with $T_{\text {eff }}:(B-V)$ and $T_{\text {eff }}:(U-V)$ calibrations, which only deviates significantly from ours in the red edge of colour axes.

Differences found with the calibration $T_{\text {eff }}:(B-V)$ based on the IRFM of Blackwell \& Lynas-Gray (1998; BL98) for Population I stars is compatible with a zero point shift amounting to $70 \mathrm{~K}$.

The calibration presented by Böhm-Vitense (1981) for stars with $z / z_{\odot}=0.01$ is in very good agreement with our calibration for $[\mathrm{Fe} / \mathrm{H}]=-2$, however strong discrepancies are found for Population I calibration.

We provide also comparison with the scale of Montegriffo et al. (1998; M98). It must be pointed out that although it is not properly a homogeneous calibration but an amalgam based on previous calibrations, it takes into account the effect of metallicity although in a rough manner. Our temperatures for solar metalicity stars are $\sim 200 \mathrm{~K}$ larger than M98 ones, however our temperatures for metal-poor stars are $\sim 150 \mathrm{~K}$ smaller,

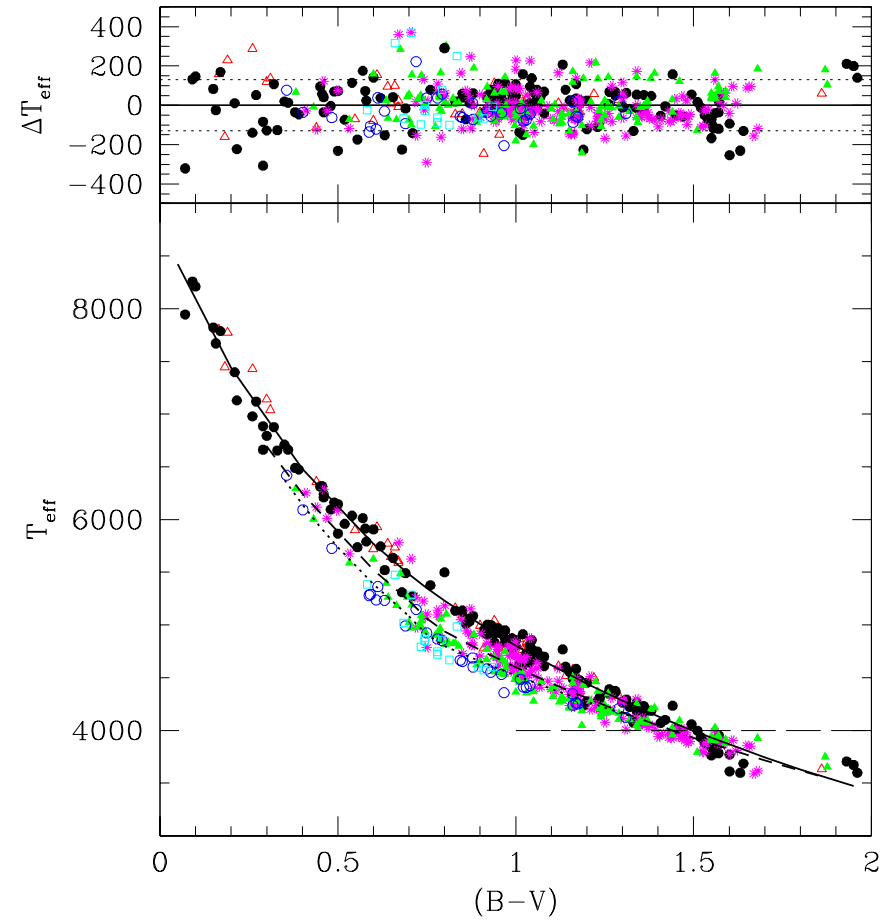

Fig. 2. $T_{\text {eff }}$ against $(B-V)$. The lines display the fit corresponding to Eqs. (3) and (4) for $[\mathrm{Fe} / \mathrm{H}]=0$ (solid line), $[\mathrm{Fe} / \mathrm{H}]=-1$ (dashed line), $[\mathrm{Fe} / \mathrm{H}]=-2($ dotted line $)$. Symbols stand for the same metallicity groups as in Fig. 1. The horizontal long-dashed line delineates the region $T_{\text {eff }} \leq 4000 \mathrm{~K}$ where temperatures derived by means of the IRFM have lower accuracy. The top panel of the figure shows the residuals of the fit

the reason for these discrepancies is unclear, but they are similar to those found when comparing calibrations of temperature against other colours in Sects. 2.2 and 2.3.

In summary, differences observed are significant and illustrate the state of the art of temperature calibrations. Two conclusions may be extracted from the above analysis, on the one hand, our semi-empirical calibrations differ from theoretical ones in a systematic manner, discrepancies ranging $\pm 5 \%$ (Figs. 3a,b). On the other hand, a better agreement is found in general with semi-empirical calibrations. In this case, differences are within $\pm 2 \%$ in the range $8000>T_{\text {eff }}>4500 \mathrm{~K}$ (Figs. 3a,c).

\section{2. $T_{\mathrm{eff}}:[\mathrm{Fe} / \mathrm{H}]:(V-R),:(V-I)$ and $:(R-I)$}

The importance of $V R I$ photometry is increasing, among other advantages, because of the better quantum efficiency of the first generation of CCD detectors in the optical-infrared wavelength range covered by these filters. Furthermore, a considerable part of the flux of red giant stars, and in general of cool stars, is emitted through $R, I$ bands. As a consequence $(V-R),(V-I)$ and $(R-I)$ colours have revealed themselves as very useful stellar temperature indicators in many investigations. Although 

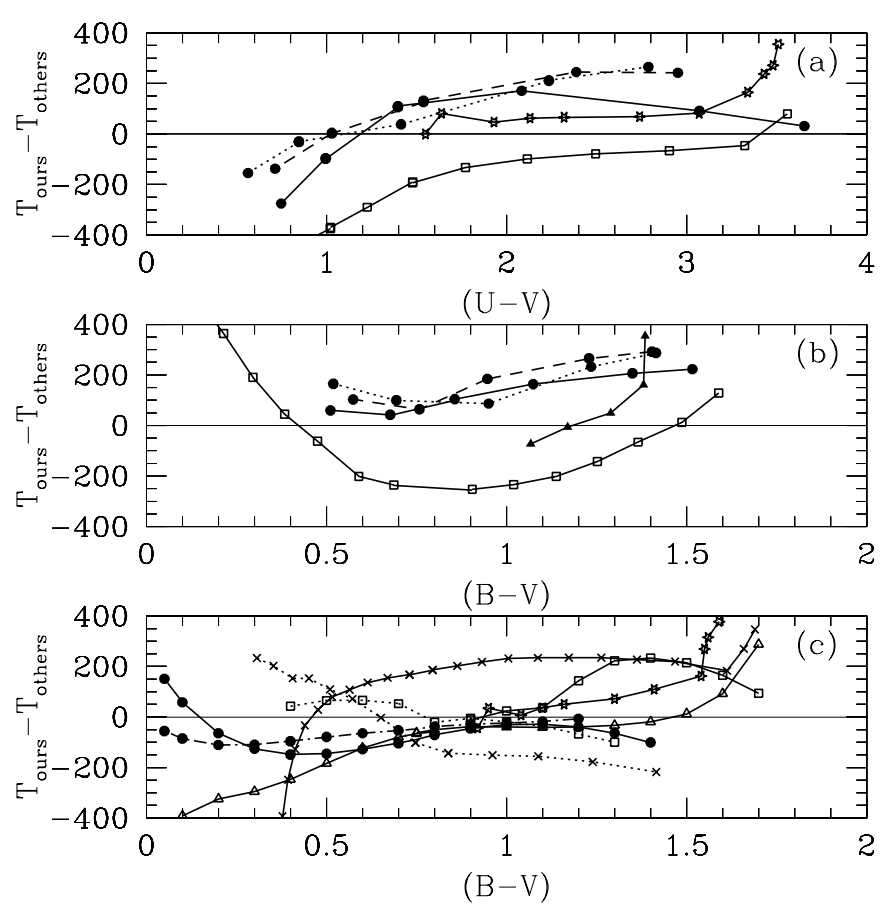

Fig. 3. Comparison between the present calibrations and several published calibrations of $T_{\text {eff }}$ against $(U-V)$ and $(B-V)$. a,b) Theoretical calibrations. Squares: calibration of Buser \& Kurucz (1992; BK92) based on Kurucz models (Solid lines: $[\mathrm{Fe} / \mathrm{H}]=0$, dashed lines: $[\mathrm{Fe} / \mathrm{H}]=-1$, dotted lines: $[\mathrm{Fe} / \mathrm{H}]=-2)$; circles: Calibration for $[\mathrm{Fe} / \mathrm{H}]=0$ of Bessel et al. (1998; BCP98) based on Kurucz models. Triangles: Calibration BCP97 based on NMARCS models. c) Empirical calibrations. Squares: Böhm-Vitense (1981) (Solid lines: $[\mathrm{Fe} / \mathrm{H}]=0$, dotted lines: $[\mathrm{Fe} / \mathrm{H}]=-2)$; Triangles: Calibration of Flower (1996); Circles: Calibrations of Blackwell \& LynasGray (1998; BL98); Crosses: Calibration of Montegriffo et al. (1998; M98) (Solid lines: metal-rich giants, dotted lines: metal-poor giants)

Cousins' system has been standardised even in the northern hemisphere, here we have adopted Johnson's system as a reference because most of the photometry of the stars in our sample is in this system. Transformations between Johnson, Cousins and many other systems are well determined by Bessell (1979) and Fernie (1983).

The fit obtained for $T_{\text {eff }}:[\mathrm{Fe} / \mathrm{H}]:(V-R)$ is shown in Table 2 (Eq. (5)); the corresponding ranges of application are shown in Table 3. We display in Fig. 4 the mean lines corresponding to Eq. (5), together with the residuals of the fit.

The mean variation $\Delta T_{\text {eff }} / \Delta(V-R)$ amounts approximately to $58 \mathrm{~K}$ per $0.01 \mathrm{mag}$ for $(V-R)<0.65$ and $16 \mathrm{~K}$ per $0.01 \mathrm{mag}$ for $(V-R)>0.65$. At constant $(V-R)$, temperature monotonically decreases with decreasing $[\mathrm{Fe} / \mathrm{H}]$. The gradient $\Delta T_{\text {eff }} / \Delta[\mathrm{Fe} / \mathrm{H}]$ shows little dependence on colour and tends to zero as $[\mathrm{Fe} / \mathrm{H}]$ decreases (saturation occurring at $[\mathrm{Fe} / \mathrm{H}] \approx-2.5)$. When using this calibration an error of $0.03 \mathrm{mag}$ in $(V-R)$ implies mean errors of
Table 3. Colour and metallicity ranges of applicability of temperature calibrations

\begin{tabular}{|c|c|c|c|c|c|}
\hline Eq. \# & Colour & Colour range & Metal & licity $\mathrm{r}$ & range \\
\hline 1 & $(U-V)$ & $0.40 \leq(U-V) \leq 1.20$ & $+0.2 \geq$ & {$[\mathrm{Fe} / \mathrm{H}]$} & $>-0.5$ \\
\hline & & $0.35 \leq(U-V) \leq 1.20$ & $-0.5 \geq$ & $\mathrm{Fe} / \mathrm{H}]$ & $>-1.5$ \\
\hline & & $0.40 \leq(U-V) \leq 1.20$ & $-1.5 \geq$ & $\mathrm{Fe} / \mathrm{H}]$ & $>-2.5$ \\
\hline & & $0.50 \leq(U-V) \leq 1.20$ & $-2.5 \geq$ & $\mathrm{Fe} / \mathrm{H}]$ & $>-3.0$ \\
\hline 2 & $(U-V)$ & $1.50 \leq(U-V) \leq 3.50$ & $+0.2 \geq$ & $\overline{\mathrm{Fe} / \mathrm{H}]}$ & $>-0.5$ \\
\hline & & $1.50 \leq(U-V) \leq 3.50$ & $-0.5 \geq$ & $\mathrm{Fe} / \mathrm{H}]$ & $>-1.5$ \\
\hline & & $1.50 \leq(U-V) \leq 3.25$ & $-1.5 \geq$ & $\mathrm{Fe} / \mathrm{H}]$ & $>-2.5$ \\
\hline 3 & $(B-V)$ & $0.20 \leq(B-V) \leq 0.80$ & $+0.2 \geq$ & $\overline{\mathrm{Fe} / \mathrm{H}]}$ & $>-0.5$ \\
\hline & & $0.35 \overline{\leq}(B-V) \leq 0.80$ & $-0.5 \geq$ & $\mathrm{Fe} / \mathrm{H}]$ & $>-1.5$ \\
\hline & & $0.35 \leq(B-V) \leq 0.80$ & $-1.5 \geq$ & $\mathrm{Fe} / \mathrm{H}]$ & $>-2.5$ \\
\hline & & $0.50 \leq(B-V) \leq 0.80$ & $-2.5 \geq$ & $\mathrm{Fe} / \mathrm{H}]$ & $>-3.0$ \\
\hline 4 & $(B-V)$ & $0.70 \leq(B-V) \leq 1.90$ & $+0.2 \geq$ & $\overline{\mathrm{Fe} / \mathrm{H}]}$ & $>-0.5$ \\
\hline & & $0.70 \leq(B-V) \leq 1.80$ & $-0.5 \geq$ & {$[\mathrm{Fe} / \mathrm{H}]$} & $>-1.5$ \\
\hline & & $0.70 \leq(B-V) \leq 1.35$ & $-1.5 \geq$ & $\mathrm{Fe} / \mathrm{H}]$ & $>-2.5$ \\
\hline & & $0.70 \leq(B-V) \leq 1.00$ & $-2.5 \geq$ & $\mathrm{Fe} / \mathrm{H}]$ & $>-3.0$ \\
\hline 5 & $(V-R)$ & $0.15 \leq(V-R) \leq 1.70$ & $+0.2 \geq$ & $\overline{\mathrm{Fe} / \mathrm{H}]}$ & $>-0.5$ \\
\hline & & $0.45 \leq(V-R) \leq 1.50$ & $-0.5 \geq$ & $\mathrm{Fe} / \mathrm{H}]$ & $>-1.5$ \\
\hline & & $0.50 \leq(V-R) \leq 1.00$ & $-1.5 \geq$ & $\mathrm{Fe} / \mathrm{H}]$ & $>-2.5$ \\
\hline & & $0.55 \leq(V-R) \leq 0.85$ & $-2.5 \geq$ & $\mathrm{Fe} / \mathrm{H}]$ & $>-3.0$ \\
\hline 6 & $(V-I)$ & $0.20 \leq(V-I) \leq 2.90$ & $+0.2 \geq$ & $\mathrm{Fe} / \mathrm{H}]$ & $>-0.5$ \\
\hline & & $0.80 \leq(V-I) \leq 2.00$ & $-0.5 \geq$ & $\mathrm{Fe} / \mathrm{H}$ & $>-1.5$ \\
\hline & & $0.85 \leq(V-I) \leq 2.20$ & $-1.5 \geq$ & $\mathrm{Fe} / \mathrm{H}]$ & $>-2.5$ \\
\hline & & $1.00 \leq(V-I) \leq 1.70$ & $-2.5 \geq$ & $\mathrm{Fe} / \mathrm{H}]$ & $>-3.0$ \\
\hline 7 & $(R-I)$ & $0.15 \leq(R-I) \leq 1.40$ & $+0.2 \geq$ & $\overline{\mathrm{Fe} / \mathrm{H}]}$ & $>-0.5$ \\
\hline & & $0.25 \leq(R-I) \leq 0.80$ & $-0.5 \geq$ & $\mathrm{Fe} / \mathrm{H}]$ & $>-1.5$ \\
\hline & & $0.35 \leq(R-I) \leq 0.70$ & $-1.5 \geq$ & $\mathrm{Fe} / \mathrm{H}]$ & $>-2.5$ \\
\hline & & $0.40 \leq(R-I) \leq 0.65$ & $-2.5 \geq$ & {$[\mathrm{Fe} / \mathrm{H}]$} & $>-3.0$ \\
\hline 8 & $(V-K)$ & $0.20 \leq(V-K) \leq 2.50$ & $+0.2 \geq$ & $\mathrm{Fe} / \mathrm{H}]$ & $>-0.5$ \\
\hline & & $1.00 \leq(V-K) \leq 2.50$ & $-0.5 \geq$ & $\mathrm{Fe} / \mathrm{H}]$ & $>-1.5$ \\
\hline & & $1.20 \leq(V-K) \leq 2.50$ & $-1.5 \geq$ & $\mathrm{Fe} / \mathrm{H}]$ & $>-2.5$ \\
\hline & & $1.70 \overline{\leq}(V-K) \overline{\leq} 2.50$ & $-2.5 \geq$ & $\mathrm{Fe} / \mathrm{H}]$ & $>-3.0$ \\
\hline 9 & $(V-K)$ & $2.00 \leq(V-K) \leq 4.90$ & $+0.2 \geq$ & $\mathrm{Fe} / \mathrm{H}]$ & $>-0.5$ \\
\hline & & $2.00 \leq(V-K) \leq 4.60$ & $-0.5 \geq$ & $\mathrm{Fe} / \mathrm{H}]$ & $>-1.5$ \\
\hline & & $2.00 \leq(V-K) \leq 3.40$ & $-1.5 \geq$ & $\mathrm{Fe} / \mathrm{H}]$ & $>-2.5$ \\
\hline & & $2.00 \leq(V-K) \leq 2.80$ & $-2.5 \geq$ & $\mathrm{Fe} / \mathrm{H}]$ & $>-3.0$ \\
\hline 10 & $\overline{(J-H)}$ & $0.00 \leq(J-H) \leq 0.90$ & $+0.2 \geq$ & {$[\mathrm{Fe} / \mathrm{H}]$} & $>-0.5$ \\
\hline & & $0.20 \leq(J-H) \leq 0.80$ & $-0.5 \geq$ & $\mathrm{Fe} / \mathrm{H}]$ & $>-1.5$ \\
\hline & & $0.30 \leq(J-H) \leq 0.70$ & $-1.5 \geq$ & $\mathrm{Fe} / \mathrm{H}]$ & $>-2.5$ \\
\hline & & $0.35 \leq(J-H) \leq 0.65$ & $-2.5 \geq$ & $\mathrm{Fe} / \mathrm{H}]$ & $>-3.0$ \\
\hline 11 & $(J-K)$ & $0.00 \leq(J-K) \leq 1.10$ & $+0.2 \geq$ & $\overline{\mathrm{Fe} / \mathrm{H}]}$ & $>-0.5$ \\
\hline & & $0.20 \leq(J-K) \leq 1.00$ & $-0.5 \geq$ & $\mathrm{Fe} / \mathrm{H}]$ & $>-1.5$ \\
\hline & & $0.30 \leq(J-K) \leq 0.90$ & $-1.5 \geq$ & $\mathrm{Fe} / \mathrm{H}]$ & $>-2.5$ \\
\hline & & $0.40 \leq(J-K) \leq 0.80$ & $-2.5 \geq$ & {$[\mathrm{Fe} / \mathrm{H}]$} & $>-3.0$ \\
\hline 12 & $\left(V-L^{\prime}\right)$ & $0.40 \leq\left(V-L^{\prime}\right) \leq 5.00$ & $+0.2 \geq$ & {$[\mathrm{Fe} / \mathrm{H}]$} & $>-0.5$ \\
\hline 13 & $(I-K)_{\mathrm{J}}$ & $0.00 \leq(I-K)_{J} \leq 1.90$ & $+0.2 \geq$ & $\mathrm{Fe} / \mathrm{H}]$ & $>-0.5$ \\
\hline & & $0.50 \leq(I-K)_{J} \leq 1.60$ & $-0.5 \geq$ & $\mathrm{Fe} / \mathrm{H}$ & $>-1.5$ \\
\hline & & $0.70 \leq(I-K)_{J} \leq 1.50$ & $-1.5 \geq$ & $\mathrm{Fe} / \mathrm{H}$ & $>-2.5$ \\
\hline & & $0.80 \leq(I-K)_{J} \leq 1.20$ & $-2.5 \geq$ & {$[\mathrm{Fe} / \mathrm{H}]$} & $>-3.0$ \\
\hline 14 & $(b-y)$ & $0.00 \leq(b-y) \leq 0.55$ & $+0.2 \geq$ & {$[\mathrm{Fe} / \mathrm{H}]$} & $>-0.5$ \\
\hline & & $0.30 \leq(b-y) \leq 0.55$ & $-0.5 \geq$ & $\mathrm{Fe} / \mathrm{H}]$ & $>-1.5$ \\
\hline & & $0.35 \leq(b-y) \leq 0.55$ & $-1.5 \geq$ & $\mathrm{Fe} / \mathrm{H}]$ & $>-2.5$ \\
\hline & & $0.40 \leq(b-y) \leq 0.55$ & $-2.5 \geq$ & $\mathrm{Fe} / \mathrm{H}$ & $>-3.0$ \\
\hline 15 & $(b-y)$ & $0.50 \leq(b-y) \leq 1.00$ & $+0.2 \geq$ & $\mathrm{Fe} / \mathrm{H}]$ & $>-0.5$ \\
\hline & & $0.50 \leq(b-y) \leq 0.90$ & $-0.5 \geq$ & $\mathrm{Fe} / \mathrm{H}]$ & $>-1.5$ \\
\hline & & $0.50 \leq(b-y) \leq 0.80$ & $-1.5 \geq$ & $\mathrm{Fe} / \mathrm{H}]$ & $>-2.5$ \\
\hline & & $0.50 \leq(b-y) \leq 0.70$ & $-2.5 \geq$ & $\mathrm{Fe} / \mathrm{H}$ & $>-3.0$ \\
\hline 16 & $(u-b)$ & $1.60 \leq(u-b) \leq 4.00$ & $+0.2 \geq$ & {$[\mathrm{Fe} / \mathrm{H}]$} & $>-0.5$ \\
\hline & & $1.60 \leq(u-b) \leq 3.70$ & $-0.5 \geq$ & $\mathrm{Fe} / \mathrm{H}$ & $>-1.5$ \\
\hline & & $1.60 \leq(u-b) \leq 3.40$ & $-1.5 \geq$ & $\mathrm{Fe} / \mathrm{H}$ & $>-2.5$ \\
\hline & & $1.60 \leq(u-b) \leq 2.60$ & $-2.5 \geq$ & {$[\mathrm{Fe} / \mathrm{H}]$} & $>-3.0$ \\
\hline
\end{tabular}

$2.2-1.1 \%$ in derived temperature. Equivalently, an error of 0.5 dex in $[\mathrm{Fe} / \mathrm{H}]$ may imply mean errors of $1.0 \%$.

The fit obtained for $T_{\text {eff }}:[\mathrm{Fe} / \mathrm{H}]:(V-I)$ is shown in Table 2 (Eq. (6)); the corresponding ranges of application are shown in Table 3.

We display in Fig. 5 the mean lines corresponding to Eq. (6), together with the residuals of the fit.

The mean variation $\Delta T_{\text {eff }} / \Delta(V-I)$ amounts approximately to $32 \mathrm{~K}$ per $0.01 \mathrm{mag}$ for $(V-I)<1.2$ and $10 \mathrm{~K}$ for $(V-I)>1.2$. When using this calibration an error of 


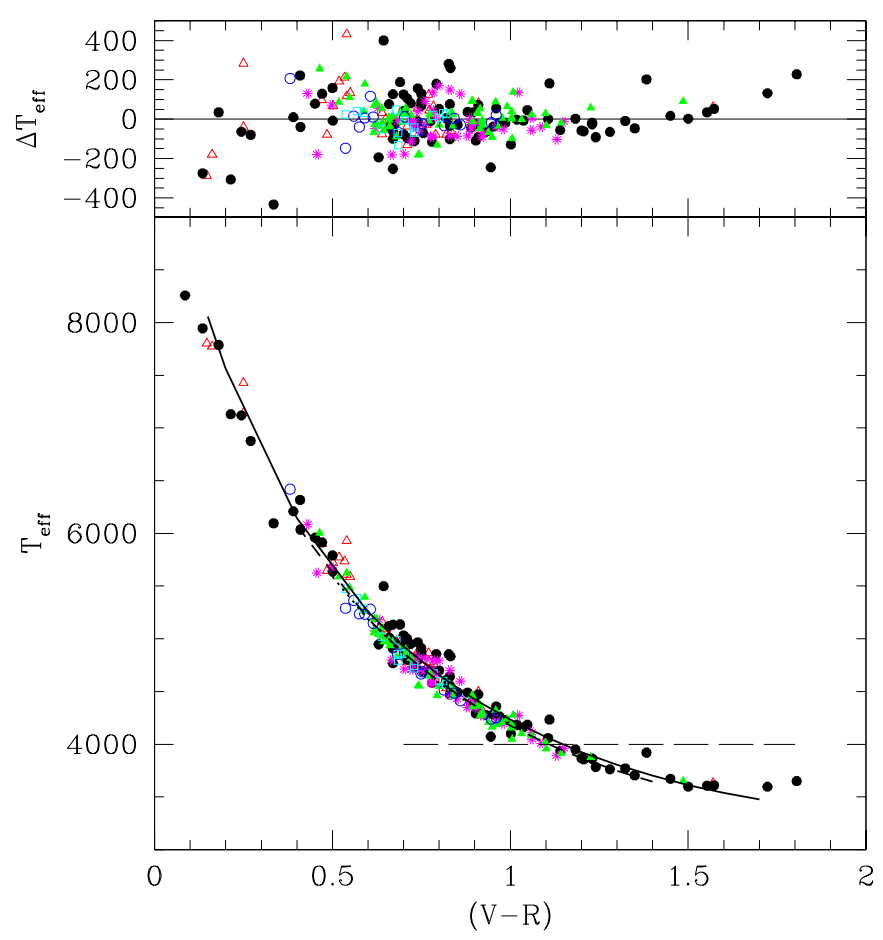

Fig. 4. $T_{\text {eff }}$ vs. $(V-R)$. The lines display the fit corresponding to Eq. (5). Symbols and lines are the same as for Fig. 2

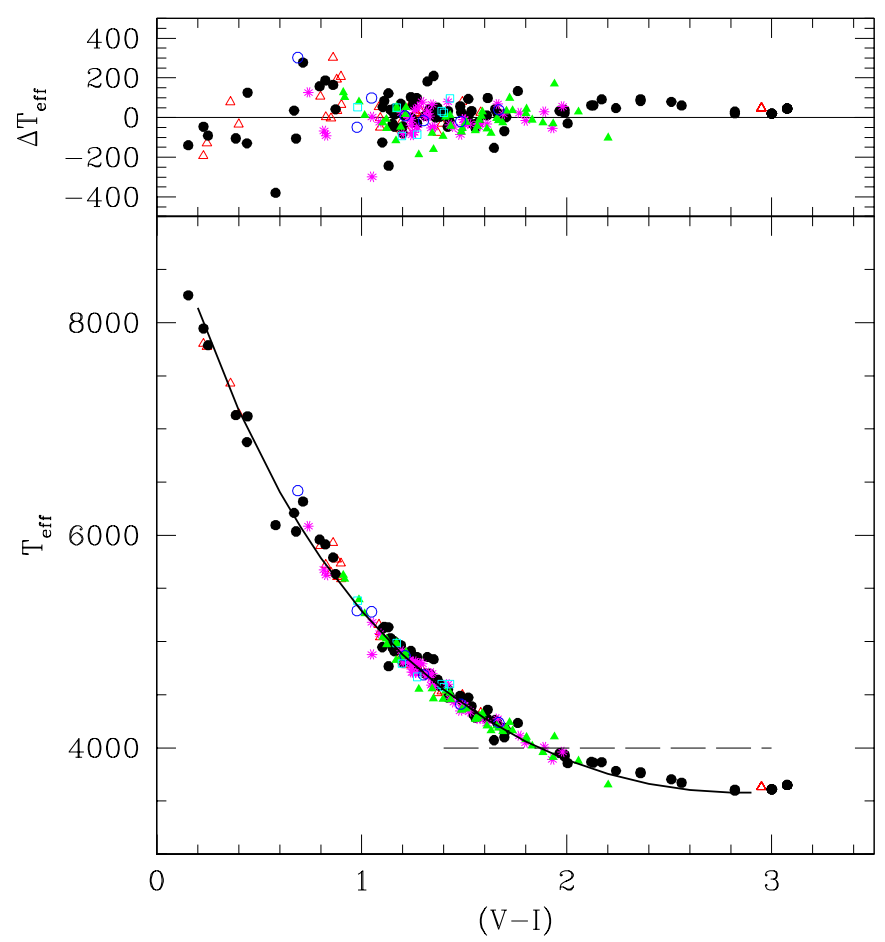

Fig. 5. $T_{\text {eff }}$ vs. $(V-I)$. The lines display the fit corresponding to Eq. (6). Symbols and lines are the same as for Fig. 2

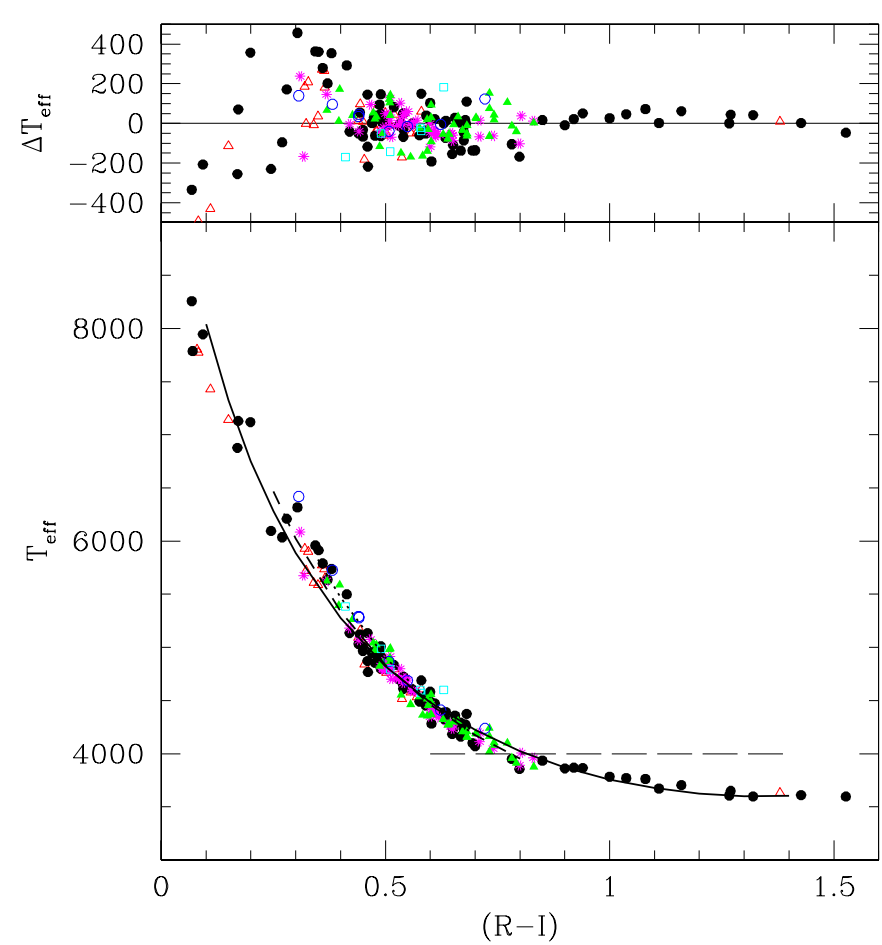

Fig. 6. $T_{\text {eff }}$ vs. $(R-I)$. The lines display the fit corresponding to Eq. (7). Symbols and lines are the same as for Fig. 2

$0.03 \mathrm{mag}$ in $(V-I)$ implies mean errors of $1.2-0.8 \%$ in temperature.

The calibration of giants' $T_{\text {eff }}$ as a function of $(V-I)$ is metallicity independent as in the case of dwarf stars (Paper III). This fact together with the relatively small value of $\Delta T_{\text {eff }} / \Delta(V-I)$ makes of $(V-I)$ an excellent temperature indicator for giants.

The fit obtained for $T_{\text {eff }}:[\mathrm{Fe} / \mathrm{H}]:(R-I)$ is shown in Table 2 (Eq. (7)); the corresponding ranges of application are shown in Table 3.

We display in Fig. 6 the mean lines corresponding to Eq. (7), together with the residuals of the fit.

The mean variation $\Delta T_{\text {eff }} / \Delta(R-I)$ amounts approximately to $70 \mathrm{~K}$ per $0.01 \mathrm{mag}$ for $(R-I)<0.6$ and $15 \mathrm{~K}$ per 0.01 mag for $(R-I)>0.6$. At constant $(R-I)$, temperature monotonically increases with decreasing $[\mathrm{Fe} / \mathrm{H}]$ in the blue range $(R-I)<0.6$, conversely it decreases with decreasing $[\mathrm{Fe} / \mathrm{H}]$ in the red range $(R-I)>0.6$. The gradient $\Delta T_{\text {eff }} / \Delta[\mathrm{Fe} / \mathrm{H}]$ depends on colour and tends to zero as $[\mathrm{Fe} / \mathrm{H}]$ decreases (saturation occurring at $[\mathrm{Fe} / \mathrm{H}]$ $\approx-3)$. When using this calibration an error of $0.03 \mathrm{mag}$ in $(R-I)$ implies mean errors of $2.7-1.1 \%$ in derived temperature. Equivalently, an error of 0.5 dex in $[\mathrm{Fe} / \mathrm{H}]$ may imply errors as large as $1.9 \%$.

In Fig. 7 we show a comparison of relations (5) and (6) with several theoretical and empirical calibrations taken from literature.

In general, theoretical calibrations (Bessell et al. (1998; BCP98) based on Kurucz models, and Buser \& Kurucz 

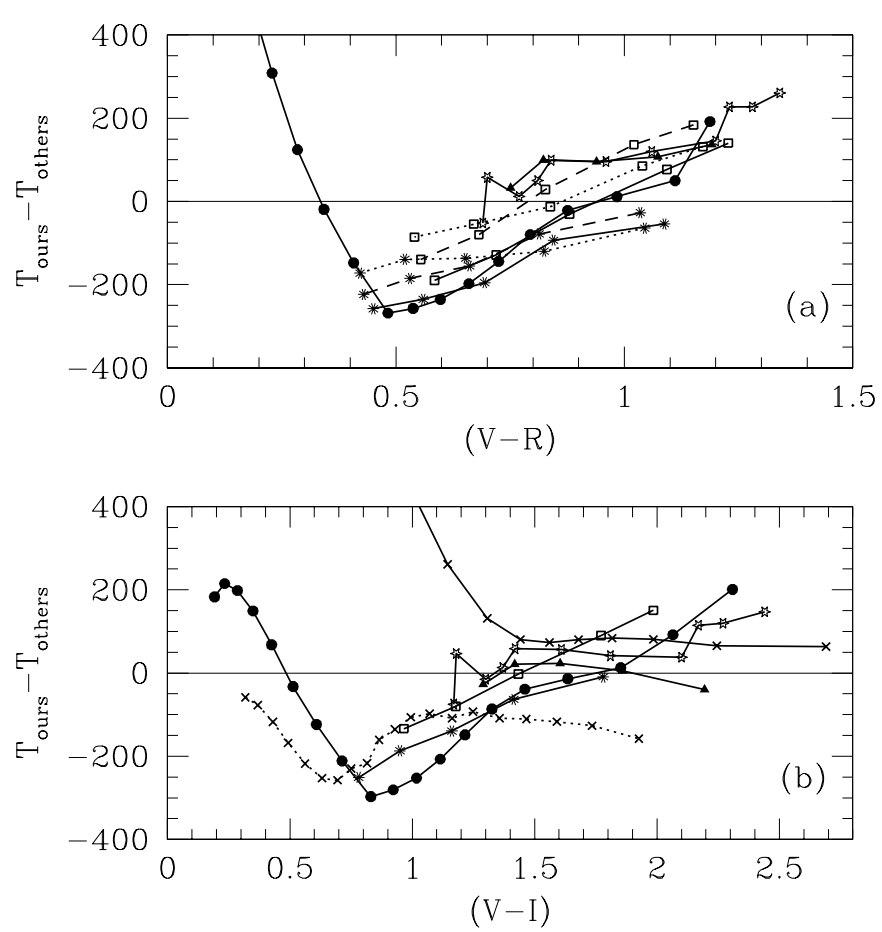

Fig. 7. a) Comparison between the present calibration and several published calibrations of $T_{\text {eff }}$ against $(V-R)$. When necessary colours have been transformed into Johnson system by using relations provided by Bessel (1979) and Fernie (1983). Stars: Calibration of Johnson (1966; J66). Circles: Calibration BCP98 based on Kurucz models. Triangles: Circles: Calibration BCP98 based on NMARCS models. Squares: calibration BK92 (Solid lines: $[\mathrm{Fe} / \mathrm{H}]=0$, dashed lines: $[\mathrm{Fe} / \mathrm{H}]=-1$, dotted lines: $[\mathrm{Fe} / \mathrm{H}]=-2)$. Asterisks: Calibration of Bell \& Gustaffson (1989; BG89) (Solid lines: $[\mathrm{Fe} / \mathrm{H}]=0$, dashed lines: $[\mathrm{Fe} / \mathrm{H}]=-1$, dotted lines: $[\mathrm{Fe} / \mathrm{H}]=-2)$. b) Comparison between the present calibration and several published calibrations of $T_{\text {eff }}$ against $(V-I)$. Symbols are the same as for a) where only $[\mathrm{Fe} / \mathrm{H}]=0$ has been considered, and Crosses: Calibration of M98 (Solid lines: metal-rich stars, dotted lines: metal-poor stars)

(1992; BK92)) show strong systematic differences both with our $T_{\text {eff }}:(V-R)$ and $T_{\text {eff }}:(V-I)$ calibrations. A much better agreement, compatible with zero-point shifts, is found with BCP98 calibration based on NMARCS models. Concerning empirical calibrations, a fairly good agreement is found with the scale of J66. However differences with Bell \& Gustaffson (1989; BG89) calibration show similar trends to those of theoretical calibrations (recall this calibration is based on IRFM temperatures corrected with synthetic colours). The comparison with M98 calibration yields again a contradictory result: On the one hand, M98 temperatures for metal-poor stars are systematically larger than ours (150 K on average), on the other, M98 temperatures for metal-rich stars are systematically smaller than ours $(75 \mathrm{~K}$ on average for $(V-I)>1.2)$, and differences increase dramatically for $(V-I)<1.2$.

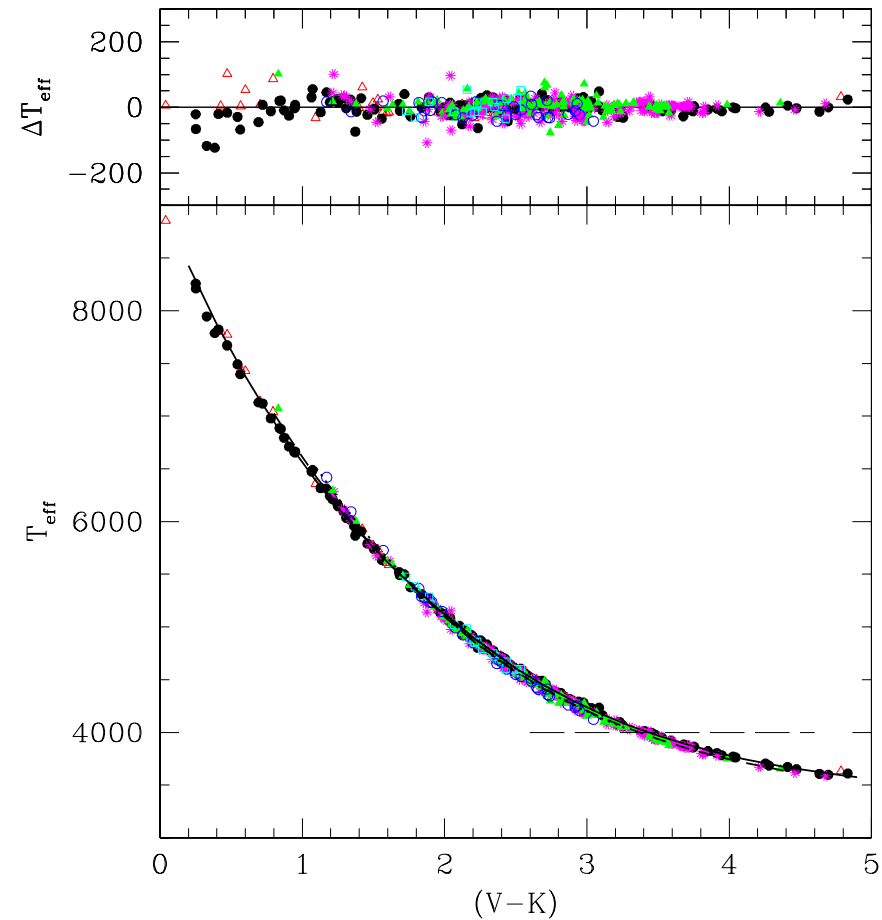

Fig. 8. $T_{\text {eff }}$ vs. $(V-K)_{\text {TCS }}$. The lines display the fit corresponding to Eqs. (8) and (9). Symbols and lines are the same as for Fig. 2. Recall that $(V-K)_{\mathrm{J}}=-0.05+1.007(V-K)_{\mathrm{TCS}}$

The difficulty of model fluxes in the $R I$ bands could account for a part of the observed differences, however it is possible that systematic errors persist in the transformations of colours of the different $V R I$ photometric systems.

2.3. $T_{\text {eff }}:[\mathrm{Fe} / \mathrm{H}]:(V-K),:(J-H),:(J-K)$ and: $\left(V-L^{\prime}\right)$

The calibration of temperature against near IR colours is of increasing interest in many studies, since these kind of relations are marginally affected by blanketing, show a small dependence on surface gravity, and IR colours are less affected by reddening than UV and optical colours. Given that a specific subprogramme of near IR photometry (Paper I) has been carried out to apply the IRFM we have adopted the TCS system as reference. Transformations of this photometric system into/from Johnson, CIT and ESO systems are provided in Paper I and references therein.

The fits obtained for $T_{\text {eff }}:[\mathrm{Fe} / \mathrm{H}]:(V-K)$ are shown in Table 2 (Eqs. (8) and (9)); the corresponding ranges of application are shown in Table 3.

In the overlapping range, a linear interpolation of relations (8) and (9) is advisable in order to avoid discontinuity.

We display in Fig. 8 the mean lines corresponding to Eqs. (8) and (9), together with the residuals of the fit.

The mean variation $\Delta T_{\text {eff }} / \Delta(V-K)$ amounts approximately to $17 \mathrm{~K}$ per $0.01 \mathrm{mag}$ for $(V-K)<2.2$ and $5 \mathrm{~K}$ 


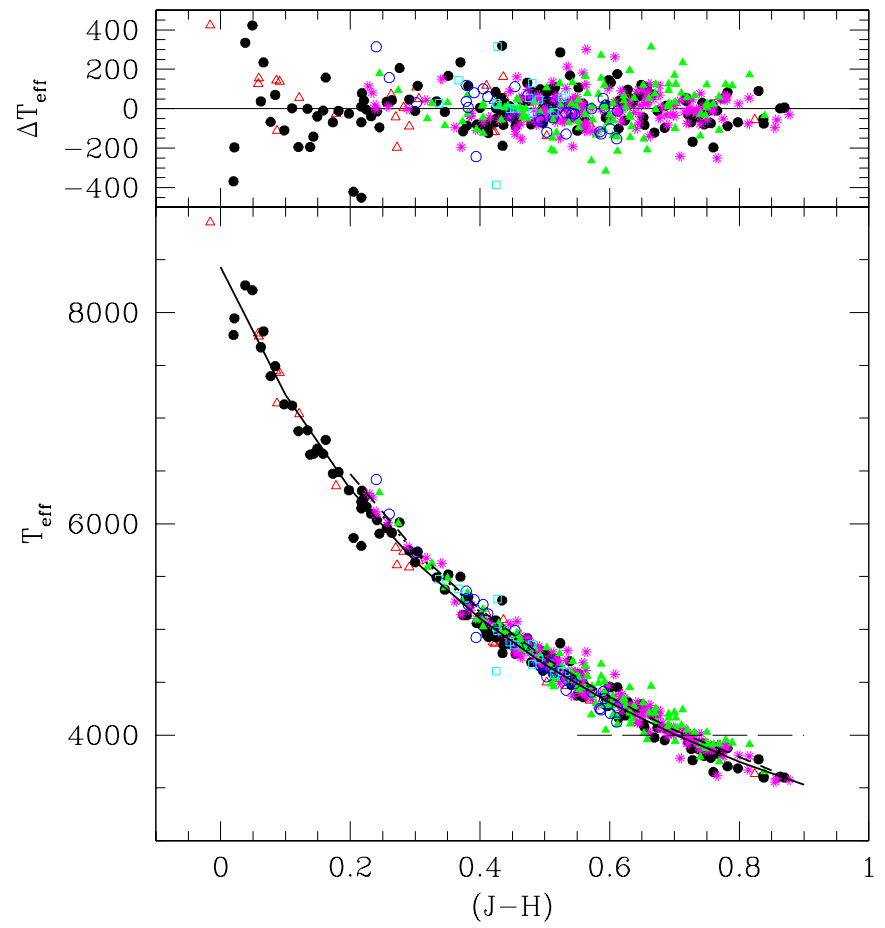

Fig. 9. $T_{\text {eff }}$ vs. $(J-H)_{\text {TCS }}$. The lines display the fit corresponding to Eq. (10). Symbols and lines are the same as for Fig. 2. Recall that $(J-H)_{\mathrm{J}}=0.011+1.062(J-H)_{\mathrm{TCS}}($ Paper I)

per $0.01 \mathrm{mag}$ for $(V-K)>2.2$. At constant $(V-K)$, temperature monotonically increases with decreasing $[\mathrm{Fe} / \mathrm{H}]$ for stars with $(V-K) \lesssim 1.8$, and monotonically decreases with decreasing $[\mathrm{Fe} / \mathrm{H}]$ for stars with $(V-K) \gtrsim 1.8$. The gradient $\Delta T_{\text {eff }} / \Delta[\mathrm{Fe} / \mathrm{H}]$ depends on colour. It is small although non-negligible, negative for $(V-K) \lesssim 1.8$ and positive for $(V-K) \gtrsim 1.8$. When using this calibration an error of 0.05 mag in $(V-K)$ implies mean errors of $1.0-0.7 \%$ in temperature. Equivalently, an error of 0.5 dex in $[\mathrm{Fe} / \mathrm{H}]$ implies at most errors of $0.7 \%$. As a consequence, $(V-K)$ is probably the best temperature indicator for giant stars.

The fit obtained for $T_{\text {eff }}:[\mathrm{Fe} / \mathrm{H}]:(J-H)$ is shown in Table 2 (Eq. (10)); the corresponding ranges of application are shown in Table 3.

We show in Fig. 9 the mean lines corresponding to Eq. (10), together with the residuals of the fit.

The mean variation $\Delta T_{\text {eff }} / \Delta(J-H)$ amounts approximately to $85 \mathrm{~K}$ per $0.01 \mathrm{mag}$ for $(J-H)<0.4$ and $32 \mathrm{~K}$ for $(J-H)>0.4$. At constant $(J-H)$, temperature monotonically increases with decreasing $[\mathrm{Fe} / \mathrm{H}]$. The gradient $\Delta T_{\text {eff }} / \Delta[\mathrm{Fe} / \mathrm{H}]$ is small and saturation occurs at $[\mathrm{Fe} / \mathrm{H}]=-2$. When using this calibration an error of $0.03 \mathrm{mag}$ in $(J-H)$ implies mean errors of $3-2.5 \%$ in temperature. Equivalently, an error of $0.5 \mathrm{dex}$ in $[\mathrm{Fe} / \mathrm{H}]$ implies at most errors of $1 \%$ in temperature.

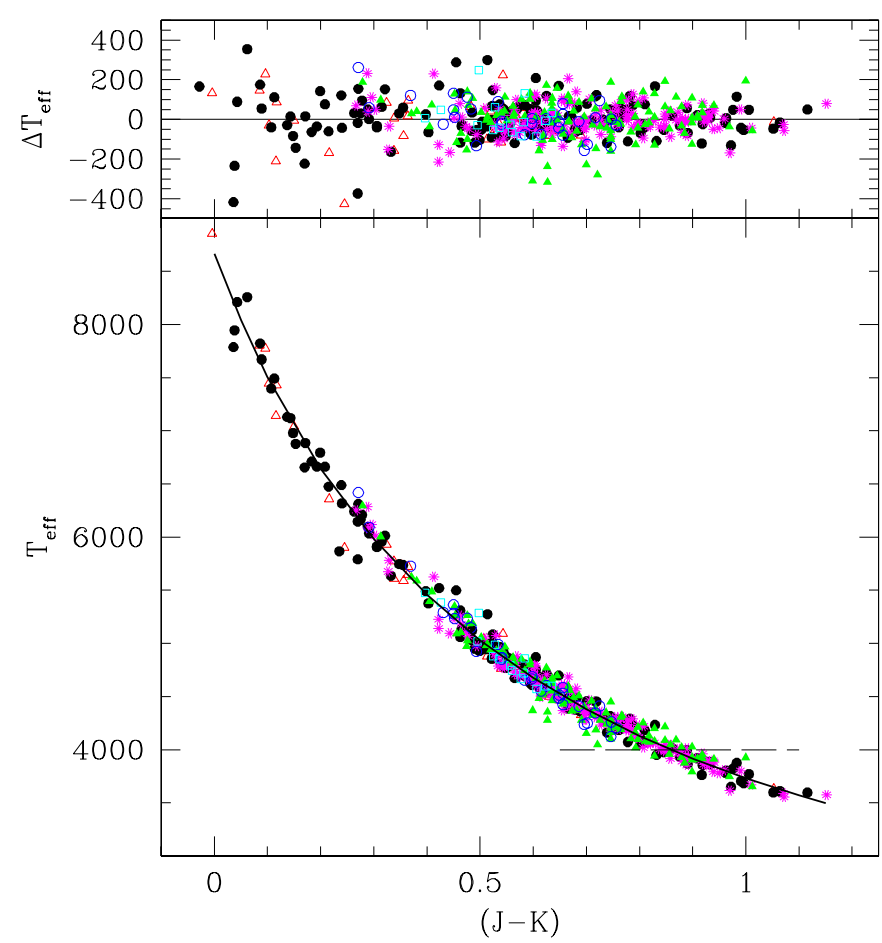

Fig. 10. $T_{\text {eff }}$ vs. $(J-K)_{\text {TCS. }}$ The lines display the fit corresponding to Eq. (11). Symbols are the same as for Fig. 2. Recall that $(J-K)_{\mathrm{J}}=-0.009+1.099(J-K)_{\mathrm{TCS}}($ Paper I)

The fit obtained for $T_{\text {eff }}:[\mathrm{Fe} / \mathrm{H}]:(J-K)$ is shown in Table 2 (Eq. (11)); the corresponding ranges of application are shown in Table 3.

We show in Fig. 10 the mean line corresponding to Eq. (11), together with the residuals of the fit.

The mean variation $\Delta T_{\text {eff }} / \Delta(J-K)$ amounts approximately to $69 \mathrm{~K}$ per $0.01 \mathrm{mag}$ for $(J-K)<0.5$ and $23 \mathrm{~K}$ for $(J-K)>0.5$. When using this calibration an error of $0.03 \mathrm{mag}$ in $(J-K)$ implies mean errors of $2.5-1.7 \%$ in temperature. Notice that the calibration of giants' $T_{\text {eff }}$ as a function of $(J-K)$ has no dependence on metallicity as in the case of dwarf stars (Paper III).

The fit obtained for $T_{\text {eff }}$ : [Fe/H]: $\left(V-L^{\prime}\right)$ is shown in Table 2 (Eq. (12)); the corresponding ranges of application are shown in Table 3.

We show in Fig. 11 the mean line corresponding to (12), together with the residuals of the fit.

The mean variation $\Delta T_{\text {eff }} / \Delta\left(V-L^{\prime}\right)$ amounts approximately to $16 \mathrm{~K}$ per $0.01 \mathrm{mag}$ for $\left(V-L^{\prime}\right)<2.4$ and $5 \mathrm{~K}$ for $\left(V-L^{\prime}\right)>2.4$. When using this calibration an error of $0.05 \mathrm{mag}$ in $\left(V-L^{\prime}\right)$ implies mean errors of $1.1-0.6 \%$ in temperature.

We show in Figs. 12a,b a comparison of relations (8) and (9) with calibrations described in previous works. As it can be appreciated, in the range $(V-K)<1.2$ differences with other empirical and theoretical calibrations for solar metallicity are contained in a band of $\pm 100 \mathrm{~K}$; In the range $(V-K)>1.2$ these differences increase as expected because of the uncertainty of bolometric flux 


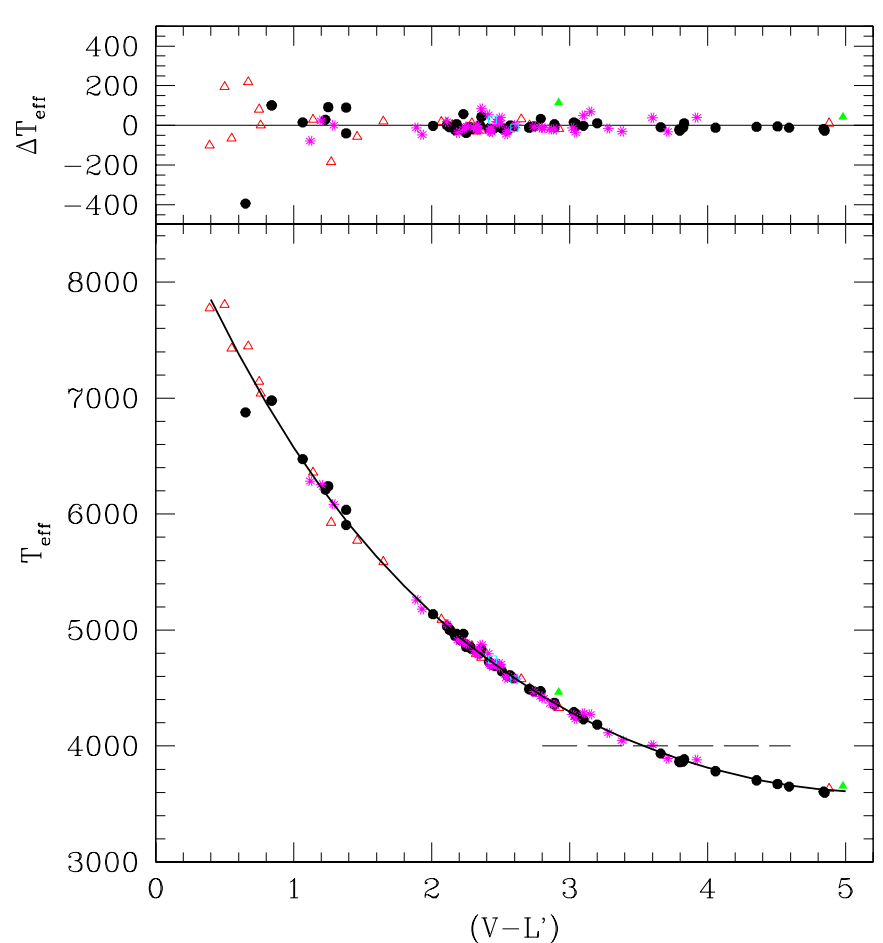

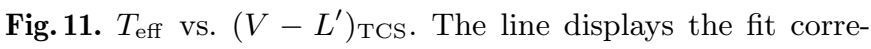
sponding to Eq. (12) Symbols are the same as for Fig. 2. Recall that $L^{\prime}=L_{\mathrm{J}}+0.04-0.016(J-K)_{\mathrm{J}}($ Paper I)

measurements for early type stars. In any case, a better level of agreement is obtained for $(V-K)$ than for other temperature indicators.

The agreement with theoretical calibrations BCP98 and BK92 is fairly good.

Concerning empirical works, the J66 calibration deviates from ours providing lower temperatures in the redder part of the colour axis.

It is remarkable, however, that there is fairly good agreement with the direct scale defined by Ridgway et al. (1980; R80) based on angular diameters measured by means of the lunar occultation method (Fig. 12b). The difference is practically a constant shift amounting to $30 \mathrm{~K}$ (our scale cooler). This fact provides a good test of the zero-point of our scale at least in the range from $3500 \mathrm{~K}$ to $4900 \mathrm{~K}$.

The two independent calibrations of temperature versus $(V-K)$ of BL98 present a slightly contradictory behaviour. One of them yields temperatures hotter than ours, and the other cooler ones. The size of the differences is small, but the reason for the inconsistency is unclear. In the range $(V-K) \gtrsim 1.5(T \lesssim 6000 \mathrm{~K})$ our calibration provides temperatures approximately $20 \mathrm{~K}$ hotter (Fig. 12b) than those of the recent calibration of Di Benedetto (1998; DB98) based on the surface brightness technique. However in the blue range, differences increase to $-350 \mathrm{~K}$ at $8500 \mathrm{~K}$.

Differences with the scale of M98, show the same behaviour observed in previous sections but the size of dis-
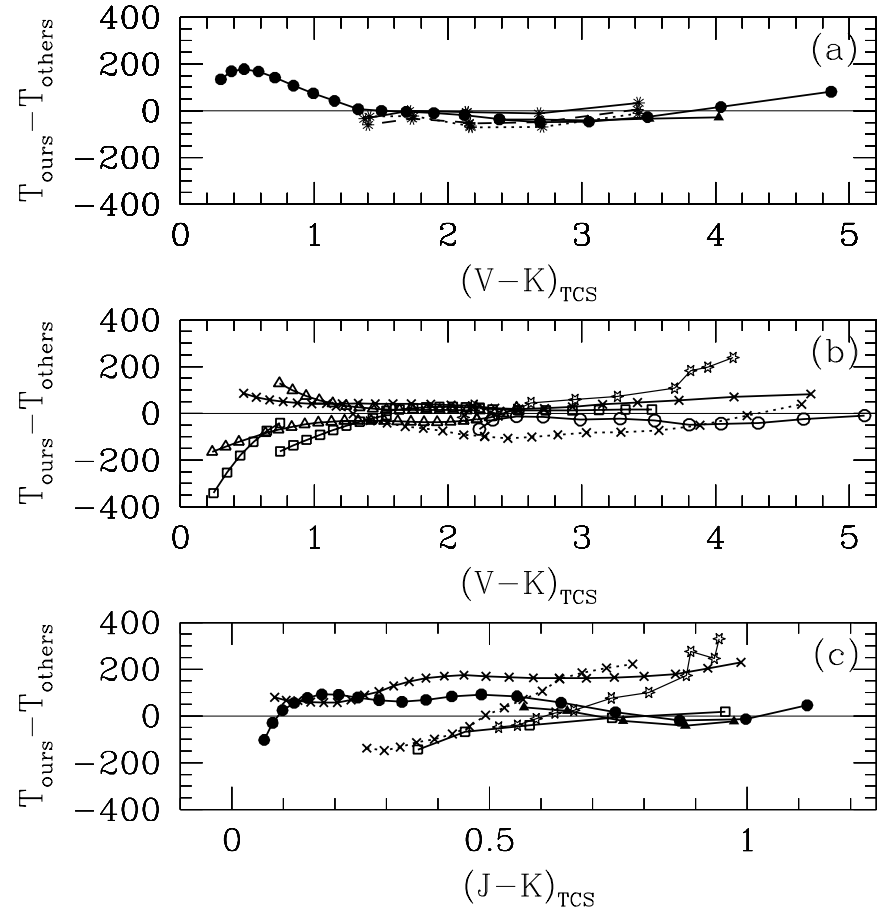

Fig. 12. Comparison between the present calibrations and several published calibrations of $T_{\text {eff }}$ against $(V-K)$ and $(J-K)$. When necessary colours have been transformed into TCS system by using relations provided in Paper I and references therein. a) Theoretical calibrations of $T_{\text {eff }}$ against $(V-K)$ : Circles BCP98 calibration based on Kurucz models; Triangles BCP98 calibration based on NMARCS models; Asterisks: BK92 calibration (Solid lines: $[\mathrm{Fe} / \mathrm{H}]=0$, dashed lines: $[\mathrm{Fe} / \mathrm{H}]=-1$, dotted lines: $[\mathrm{Fe} / \mathrm{H}]=-2)$. b) Empirical calibrations of $T_{\text {eff }}$ against $(V-K)$ : Stars: J66 calibration; Circles: Direct calibration of Ridgway et al. 1980 (R80); Triangles: Blackwell \& Lynas-Gray (1998; BL98); Squares: Di Benedetto (1998; DB98) (discontinuities observed in the line of differences are intrinsic to DB98 calibration). Crosses: M98 calibration (Solid lines: metal-rich giants, dotted lines: metal-poor giants). c) Calibrations of $T_{\text {eff }}$ against $(J-K)$ : Stars: J66 calibration; Circles BCP98 calibration based on Kurucz models; Triangles BCP98 calibration based on NMARCS models; Squares: BG89 calibration; Crosses: M98 calibration (Solid lines: metal-rich giants, dotted lines: metal-poor giants)

crepancies is somewhat small. Since M98 scale is calibrated versus optical CCD and IR array photometry, differences found could be caused by the uncertainties affecting the photometric calibration of these kind of data.

In Fig. 12c we show the comparison of our calibration $T_{\text {eff }}:(J-K)$ with other published scales. Differences are slightly larger than in the case of $T_{\text {eff }}$ : $(V-K)$ probably due to the shrinkage of the colour axis. 


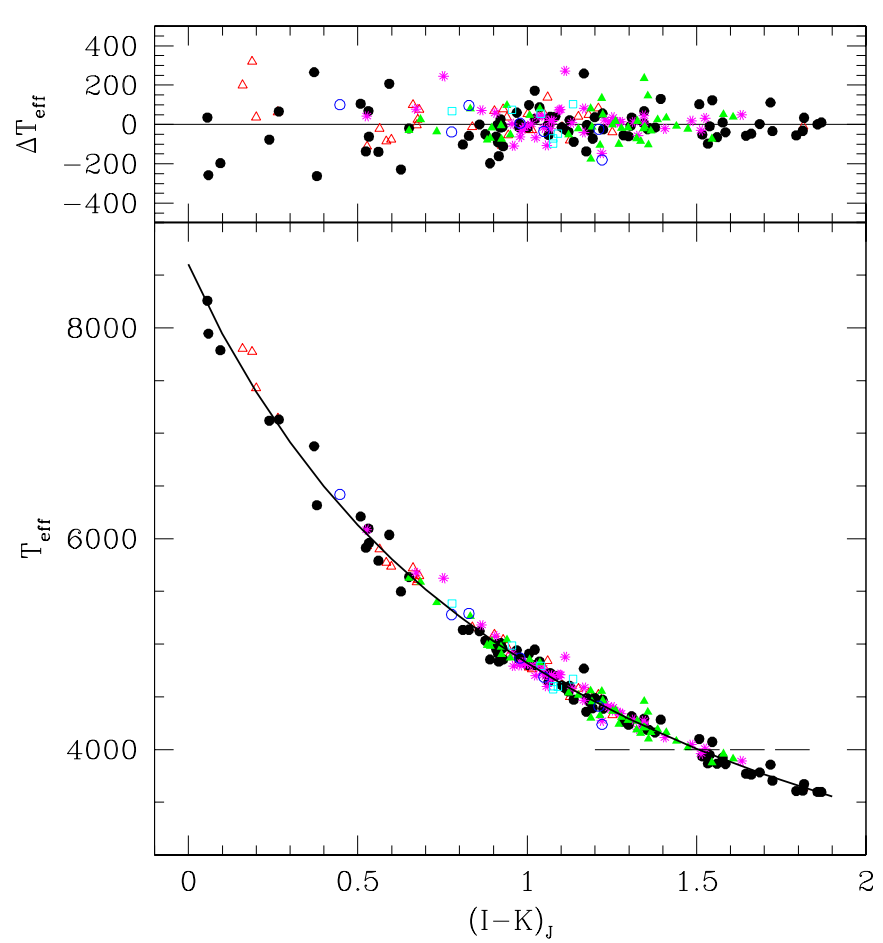

Fig. 13. $T_{\text {eff }}$ vs. $(I-K)_{J}$. The lines display the fit corresponding to Eq. (13). Symbols are the same as for Fig. 2

\section{4. $T_{\text {eff }}:[\mathrm{Fe} / \mathrm{H}]:(I-K)_{J}$}

We provide the calibration of temperature against colour $(I-K)_{\mathrm{J}}{ }^{1}$ for its interest in the study of late type giants.

The fit obtained for $T_{\text {eff }}:[\mathrm{Fe} / \mathrm{H}]:(I-K)_{\mathrm{J}}$ is shown in Table 2 (Eq. (13)); the corresponding ranges of application are shown in Table 3.

We show in Fig. 13 the mean line corresponding to (13), together with the residuals of the fit.

The mean variation $\Delta T_{\text {eff }} / \Delta(I-K)_{\mathrm{J}}$ amounts approximately to $39 \mathrm{~K}$ per $0.01 \mathrm{mag}$ for $(I-K)_{\mathrm{J}}<0.8$ and $15 \mathrm{~K}$ for $(I-K)_{\mathrm{J}}>0.8$. When using this calibration an error of $0.03 \mathrm{mag}$ in $(I-K)_{\mathrm{J}}$ implies mean errors of $1.5-1.0 \%$ in temperature. As in the case of $(V-I)$ and $(J-$ $K)$, this colour is metallicity independent as temperature indicator.

\section{5. $T_{\mathrm{eff}}:[\mathrm{Fe} / \mathrm{H}]:(b-y)$ and $:(u-b)$}

Although Strömgren system was not specifically tailored to study giant stars, its use has been extended to this class of luminosity, with internal accuracy $\lesssim 0.02 \mathrm{mag}$, both for field stars (e.g. Anthony-Twarog \& Twarog 1994) and for globular cluster stars (e.g. Davis Philip 1996). For this reason we present temperature calibrations based on colours of this system.

\footnotetext{
${ }^{1}$ We have adopted here $(I-K)$ in Johnson system, emphasizing it by adopting the subindex $J$.
}

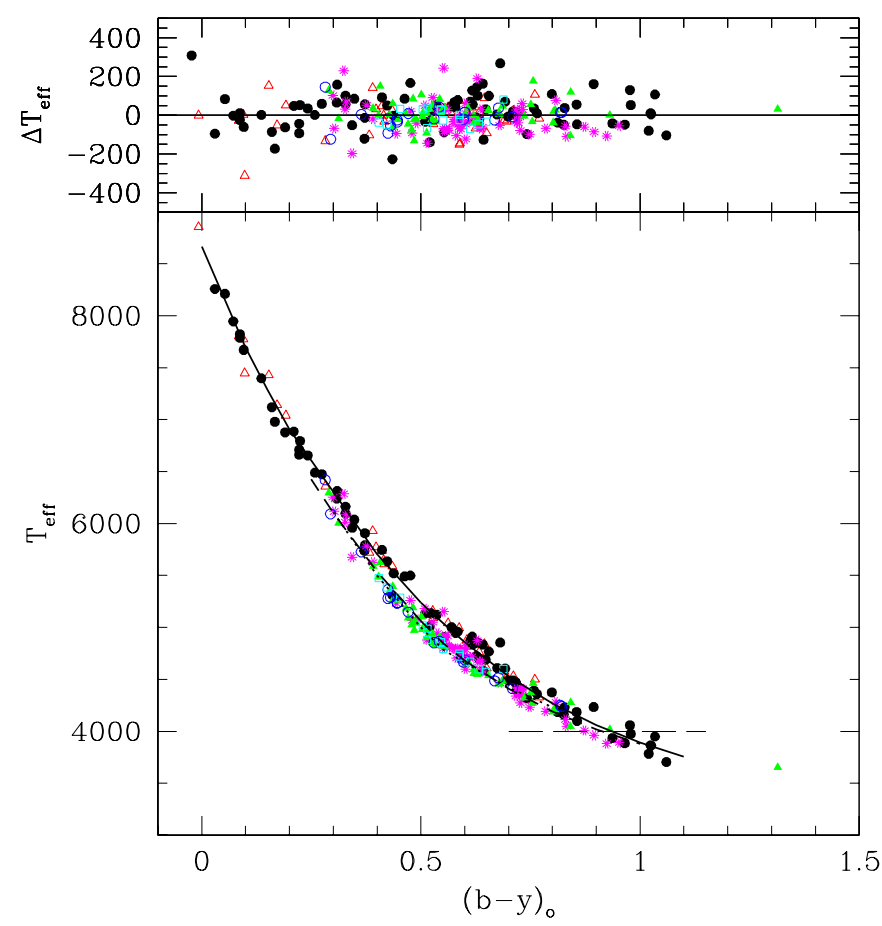

Fig. 14. $T_{\text {eff }}$ vs. $(b-y)$. The lines display the fit corresponding to Eqs. (14) and (15). Symbols and lines are the same as for Fig. 2

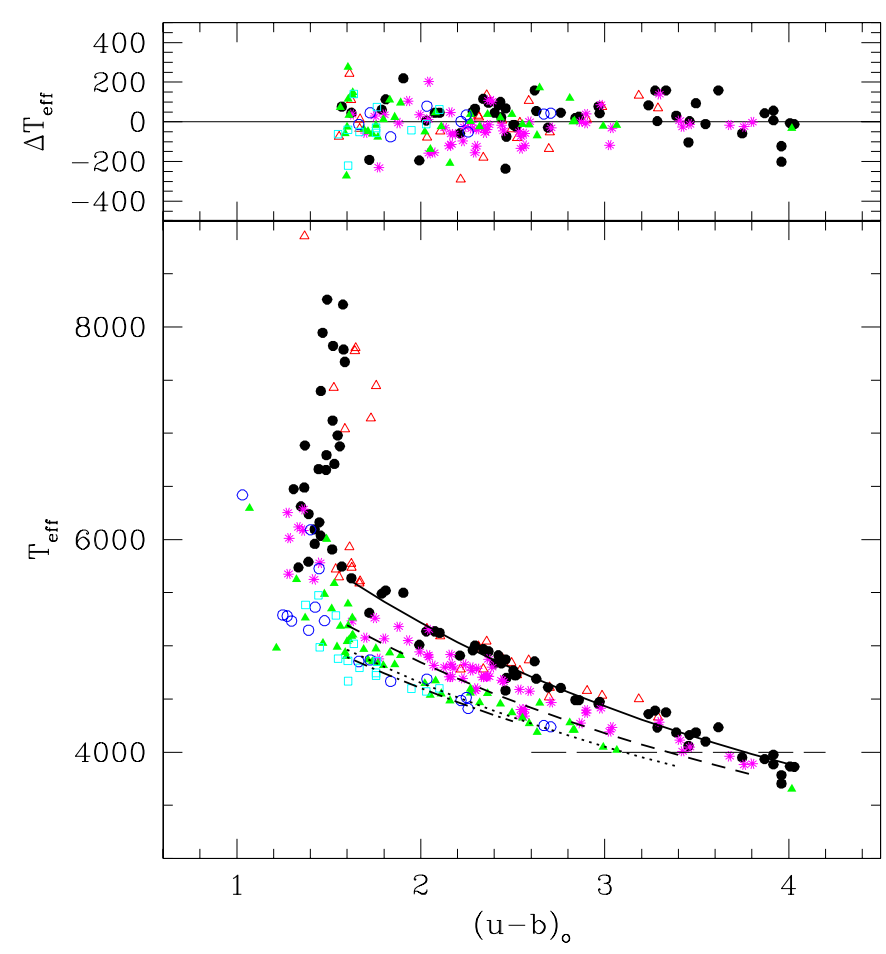

Fig. 15. $T_{\text {eff }}$ vs. $(u-b)$. The lines display the fit corresponding to Eq. (16). Symbols and lines are the same as for Fig. 1 


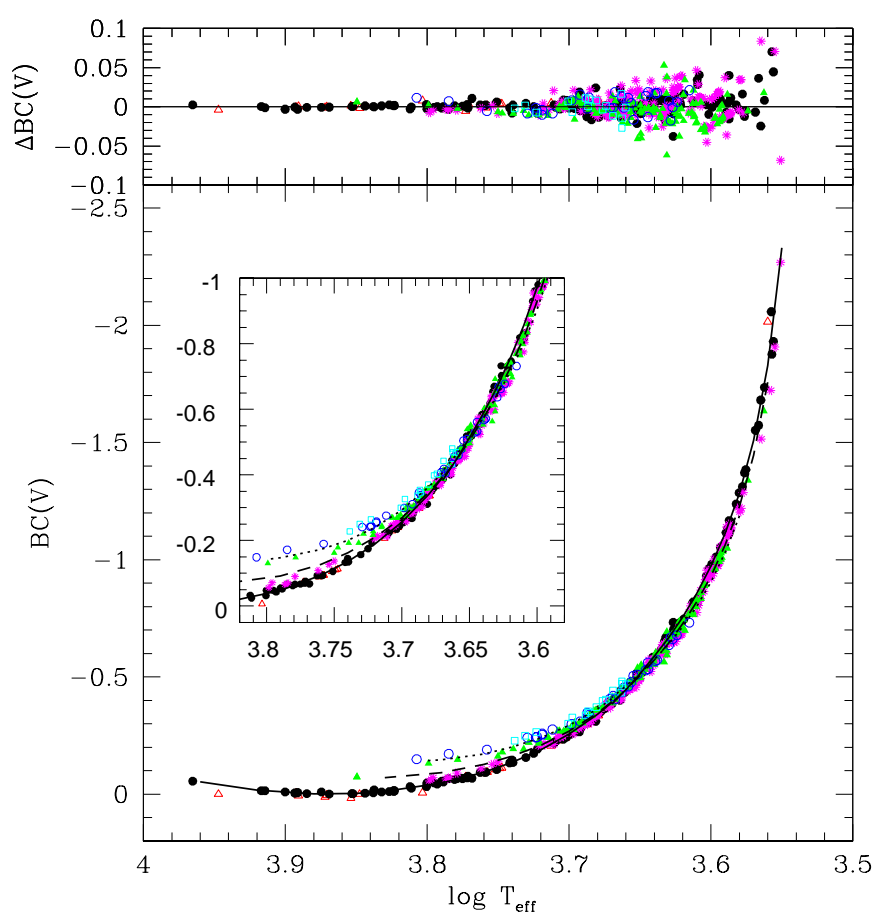

Fig. 16. $T_{\text {eff }}$ vs. BC(V). The lines display the fit corresponding to Eqs. (17) and (18). Symbols and lines are the same as for Fig. 2. The small box inside the graph contains a detailed view of the effect of metallicity on bolometric correction

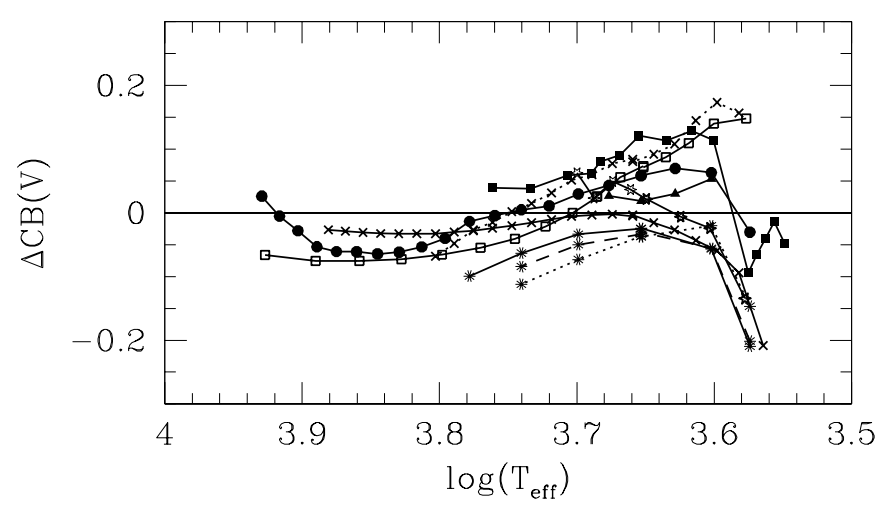

Fig. 17. Comparison between the present calibration of the bolometric correction to $V$ and several theoretical and empirical calibrations previously published. Stars: J66 calibration; Circles: BCP98 calibration based on Kurucz models; Triangles BCP98 calibration based on NMARCS models; Asterisks: BK92 calibration (Solid lines: $[\mathrm{Fe} / \mathrm{H}]=0$, dashed lines: $[\mathrm{Fe} / \mathrm{H}]=-1$, dotted lines: $[\mathrm{Fe} / \mathrm{H}]=-2)$; Crosses: M98 calibration (Solid lines: metal-rich giants, dotted lines: metalpoor giants); Open squares: F96 calibration; Full squares: Calibration of Di Benedetto (1993)
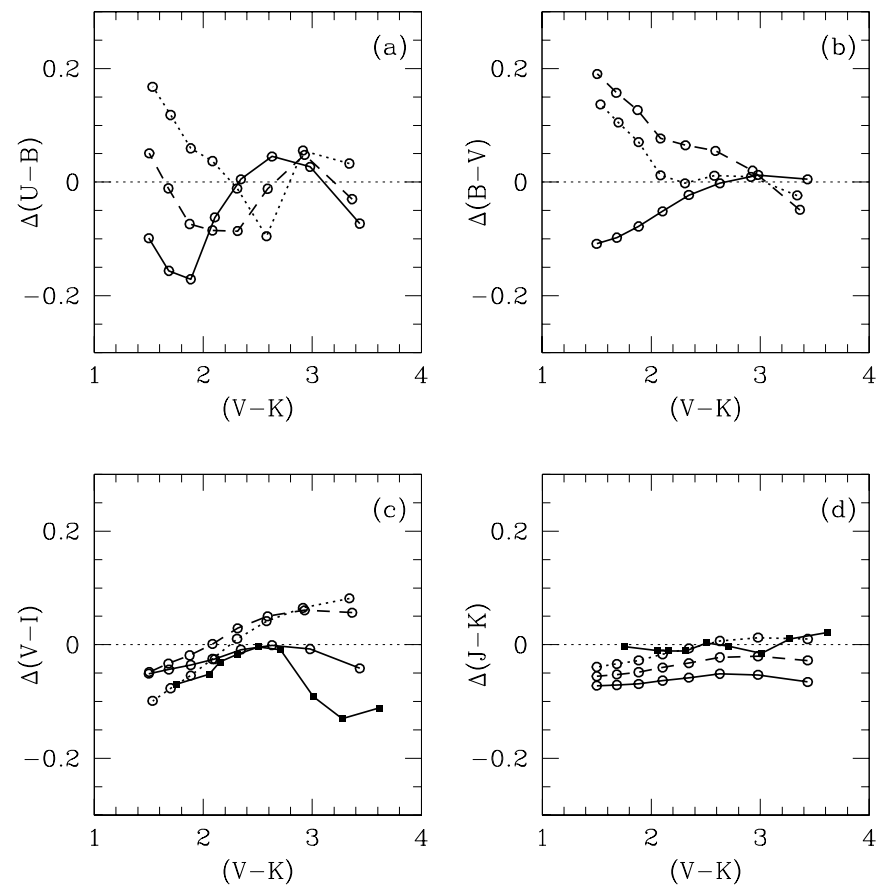

Fig. 18. Comparison between intrinsic broad band colours of giant stars derived in the present work (Table 2) and calibrations of von Braun et al. (1998) (circles), and Bessell \& Brett (1988) (squares). The difference $\Delta$ means our colours minus theirs. Solid lines: $[\mathrm{Fe} / \mathrm{H}]=0$, dashed lines: $[\mathrm{Fe} / \mathrm{H}]=-1$, dotted lines: $[\mathrm{Fe} / \mathrm{H}]=-2$
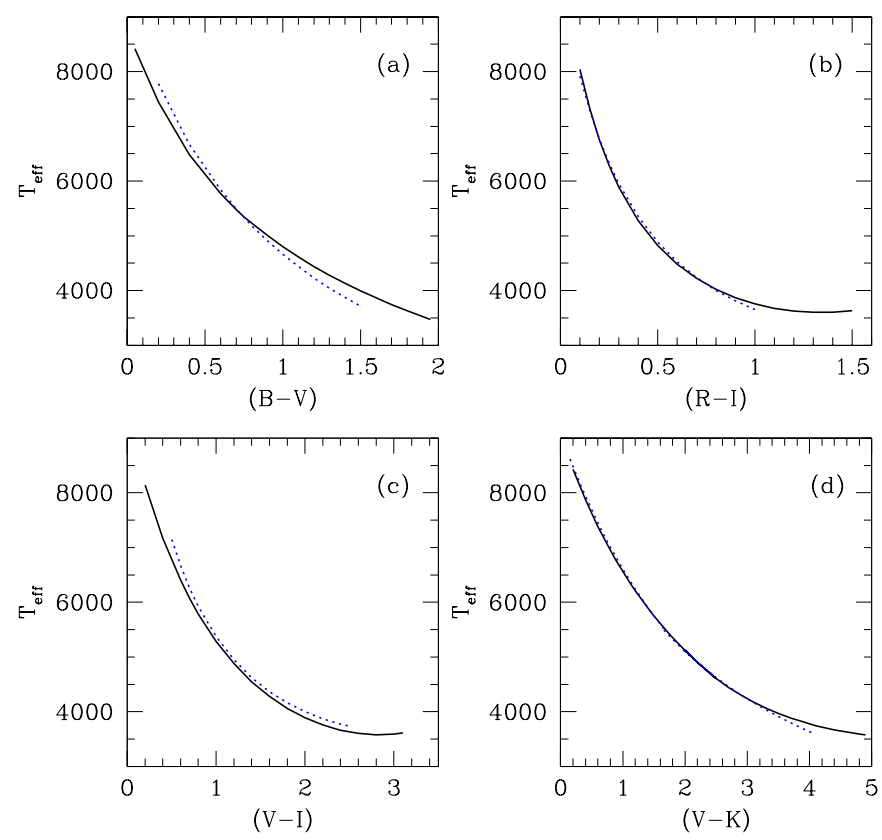

Fig. 19. Comparison between color-temperature calibration of giant stars derived in the present work (solid line) and calibrations of main sequence stars of Paper III (dotted line). Solar metallicity has been considered 
The fits obtained for $T_{\text {eff }}:[\mathrm{Fe} / \mathrm{H}]:(b-y)$ are shown in Table 2 (Eqs. (14) and (15)); the corresponding ranges of application are shown in Table 3.

In the range of colour $0.5 \leq(b-y) \leq 0.55$ a linear interpolation of Eqs. (14) and (15) provides a good fit avoiding discontinuity. We show in Fig. 14 the mean lines corresponding to (14) and (15), together with the residuals of the fit.

The mean variation $\Delta T_{\text {eff }} / \Delta(b-y)$ amounts approximately to $62 \mathrm{~K}$ per $0.01 \mathrm{mag}$ for $(b-y)<0.45$ and $26 \mathrm{~K}$ per $0.01 \mathrm{mag}$ for $(b-y)>0.45$. At constant $(b-y)$, temperature monotonically decreases with decreasing $[\mathrm{Fe} / \mathrm{H}]$. The gradient $\Delta T_{\text {eff }} / \Delta[\mathrm{Fe} / \mathrm{H}]$ depends on colour and tends to zero as $[\mathrm{Fe} / \mathrm{H}]$ decreases (saturation occurring at $[\mathrm{Fe} / \mathrm{H}] \approx-2.5)$. When using this calibration an error of $0.02 \mathrm{mag}$ in (b-y) implies mean errors of $1.5-0.8 \%$ in temperature. Equivalently, an error of $0.5 \operatorname{dex}$ in $[\mathrm{Fe} / \mathrm{H}]$ implies mean errors ranging $0.5-1.9 \%$.

The fit obtained for $T_{\text {eff }}:[\mathrm{Fe} / \mathrm{H}]:(u-b)$ (considering only stars under $6000 \mathrm{~K}$ ) is shown in Table 2 (Eq. (16)); the corresponding ranges of application are shown in Table 3. We show in Fig. 15 the mean lines corresponding to (16), together with the residuals of the fit.

The mean variation $\Delta T_{\text {eff }} / \Delta(u-b)$ amounts approximately to $8 \mathrm{~K}$ per $0.01 \mathrm{mag}$. At constant $(u-b)$ temperature monotonically decreases with decreasing $[\mathrm{Fe} / \mathrm{H}]$. The gradient $\Delta T_{\text {eff }} /[\mathrm{Fe} / \mathrm{H}]$ depends slightly on colour and diminishes as $[\mathrm{Fe} / \mathrm{H}]$ decreases as expected from atmospheres theory. The value of saturation is out of the range of the present calibration, although extrapolation provides $[\mathrm{Fe} / \mathrm{H}] \approx-3.5$. When using this calibration an error of $0.03 \mathrm{mag}$ in $(u-b)$ implies a mean error of $0.5 \%$ in temperature. Equivalently, an error of $0.5 \mathrm{dex}$ in $[\mathrm{Fe} / \mathrm{H}]$ implies mean errors of $4.2-1.5 \%$.

\section{Bolometric correction of giant stars}

Given the utility of $\mathrm{BC}(\mathrm{V})$ for the transformation of the luminosity axis of theoretical isochrones into observational $M_{V}$ of colour-magnitude diagrams, we provide here its calibration.

$$
\begin{gathered}
\mathrm{BC}(\mathrm{V})=\frac{-5.53110^{-2}}{X}-0.6177+4.420 X \\
-2.669 X^{2}+0.6943 X[\mathrm{Fe} / \mathrm{H}] \\
-0.1071[\mathrm{Fe} / \mathrm{H}]-8.61210^{-3}[\mathrm{Fe} / \mathrm{H}]^{2}, \quad(17) \\
\sigma(\mathrm{BC}(\mathrm{V}))=0.024, \quad(285 \text { stars }) \\
3.50 \leq \log \left(T_{\text {eff }}\right) \leq 3.67 \text { for }+0.2 \geq[\mathrm{Fe} / \mathrm{H}]>-0.5, \\
3.56 \leq \log \left(T_{\text {eff }}\right) \leq 3.67 \text { for }-0.5 \geq[\mathrm{Fe} / \mathrm{H}]>-1.5, \\
3.58 \leq \log \left(T_{\text {eff }}\right) \leq 3.67 \text { for }-1.5 \geq[\mathrm{Fe} / \mathrm{H}]>-2.5, \\
3.61 \leq \log \left(T_{\text {eff }}\right) \leq 3.67 \text { for }-2.5 \geq[\mathrm{Fe} / \mathrm{H}]>-3.0 . \\
\mathrm{BC}(\mathrm{V})=\frac{-9.93010^{-2}}{X}+2.88710^{-2}+2.275 X \\
-4.425 X^{2}+0.3505 X[\mathrm{Fe} / \mathrm{H}]
\end{gathered}
$$

$$
\begin{gathered}
-5.55810^{-2}[\mathrm{Fe} / \mathrm{H}]-5.37510^{-3}[\mathrm{Fe} / \mathrm{H}]^{2}, \\
\sigma(\mathrm{BC}(\mathrm{V}))=0.009, \quad(307 \text { stars })
\end{gathered}
$$

$3.65 \leq \log \left(T_{\text {eff }}\right) \leq 3.96$ for $+0.2 \geq[\mathrm{Fe} / \mathrm{H}]>-0.5$,

$3.65 \leq \log \left(T_{\text {eff }}\right) \leq 3.83$ for $-0.5 \geq[\mathrm{Fe} / \mathrm{H}]>-1.5$,

$3.65 \leq \log \left(T_{\text {eff }}\right) \leq 3.80$ for $-1.5 \geq[\mathrm{Fe} / \mathrm{H}]>-2.5$,

$3.65 \leq \log \left(T_{\text {eff }}\right) \leq 3.74$ for $-2.5 \geq[\mathrm{Fe} / \mathrm{H}]>-3.0$.

where $X=\log \left(T_{\text {eff }}\right)-3.52$. As the distribution of residuals show, this functional expansion is adequate to fit the singular behaviour of $\mathrm{BC}(\mathrm{V})$ with temperature. However, the extrapolation of the present calibration is unsafe, especially in the range of low temperatures.

The following stars departed more than $2.5 \sigma$ in either of the final fits:

BS4902 (2.8), BD-180271 (2.5), BS2286 (7.3), BS7523 (5.2),47Tuc-1421 (2.8), 47Tuc-3512 (3.4), 47Tuc-7320 (4.8), 47Tuc-1414 (2.5), 47Tuc-1518 (2.6), 47Tuc-4411 (3.1), 47Tuc-6509 (3.9), M71-B (2.9), HD 088609 (3.1), BS8930 (2.6), HD 171496 (4.7), BS3547 (2.8). We show in Fig. 16 the mean lines corresponding to (17) and (18), together with the residuals of the fit.

In the range $T_{\text {eff }}<4500$ relation (18) is not suited to deriving accurate bolometric corrections since on the one hand the dispersion of the fit is around $2.5 \%$ (0.025 mag) due to the fact that cool temperatures have greater internal errors, and on the other, the variation of $\mathrm{BC}(\mathrm{V})$ with $\log \left(T_{\text {eff }}\right)$ is very steep. A noticeable feature of the present scale is the significant variation of the bolometric correction with metallicity, especially in the range of higher temperatures (this point is emphasized in Fig. 16). Although a systematic bias in the data can never be completely discarded, the size of the effect found is not compatible either with photometric errors, or with the internal uncertainties in temperatures.

In Fig. 17, we show the comparison between the present calibration and several theoretical and empirical calibrations previously published. Differences in the zero-point caused by the adopted bolometric correction of the sun have been taken into account. In the range $8000 \mathrm{~K} \geq T_{\text {eff }} \geq 6000 \mathrm{~K}$ our scale is systematically over the other scales considered $(\sim 0.05 \mathrm{mag})$, in the range $6000 \mathrm{~K} \geq T_{\text {eff }} \geq 4000 \mathrm{~K}$ differences are symmetrically distributed in a band of $\pm 0.10 \mathrm{mag}$. These differences show the existence of essential problems in deriving bolometric corrections. In the case of empirical calibrations the possible causes of discrepancies might be the absolute flux calibration, the way of fixing the zero-point and an insufficient discrimination of the metallicity effect. In the case of theoretical calibrations, the possible causes are drawbacks in the model atmospheres and/or difficulties in the synthesis of colours.

\section{The intrinsic colours of giant stars}

In this section we present the intrinsic colours of giant stars. Tables 5, 6 and 7 have been obtained by means 
Table 4. Stars discarded in the final loops of the fit procedure. The values in parentheses refer to the number of standard deviations by which a given star departs from the corresponding fit

\begin{tabular}{|c|c|c|c|}
\hline Star & Discarded colours & Star & Discarded colours \\
\hline $\mathrm{BD}+042466$ & $(2.5 B V)$ & SAO078681 & $(2.6 V R),(2.9 V I)$ \\
\hline $\mathrm{BD}+092860$ & $(3.0 \mathrm{BV}),(2.6 J H)$ & SAO089549 & $(3.2 V I),(3.4 I K)$ \\
\hline $\mathrm{BD}-180271$ & $(3.6 V K)$ & SAO105082 & $(2.7 V I)$ \\
\hline BS0219 & $(2.6 J K),(2.7 J H)$ & SAO144233 & $(2.6 R I)$ \\
\hline BS0343 & $(2.6 R I)$ & SAO152644 & $(2.8 R I)$ \\
\hline BS0911 & $(3.32 B V),(3.3 u b)$ & SAO158392 & $(2.5 R I)$ \\
\hline BS2002 & $(2.8 V R),(2.8 V I)$ & SAO33445 & $(2.9 B V)$ \\
\hline BS2990 & $(3.4$ by $)$ & M67-117 & $(2.6 J H),(2.8 J K)$ \\
\hline BS3547 & $(2.9 V K)$ & M67-231 & $(2.7 \mathrm{JH})$ \\
\hline BS3550 & $(2.6$ by $)$ & 47 Tuc -1414 & $(2.5 U V),(2.6 V K)$ \\
\hline BS4336 & $(2.6 V R),(3.0 J H),(3.9 U V)$ & 47Tuc-2416 & $(2.5 V K)$ \\
\hline BS5301 & $(3.1 B V)$ & 47 Tuc -4417 & $(2.6 V K)$ \\
\hline BS5480 & $(2.9 V R)$ & 47 Tuc -6502 & $(4.2 V K)$ \\
\hline BS6469 & $(3.1$ by $)$ & 47 Tuc -7320 & $(2.7 U V),(3.0 \mathrm{JK}),(3.8 \mathrm{JH})$ \\
\hline BS7322 & $(2.7 V K)$ & M71-18 & $(3.2 \mathrm{BV})$ \\
\hline BS7633 & $(2.6 V R)$ & M71-75 & $(3.0 \mathrm{JH})$ \\
\hline BS7636 & $\left(3.5 V L^{\prime}\right)$ & M71-A5 & $(3.5 U V)$ \\
\hline BS7776 & $(3.7 V I),(3.4 I K)$ & $\mathrm{M} 71-\mathrm{N}$ & $(3.9 U V)$ \\
\hline BS7928 & $\left(4.6 V L^{\prime}\right)$ & NGC $1261-81$ & $(2.8 J K)$ \\
\hline BS8649 & $(3.3 V R),(2.9 \mathrm{VI})$ & NGC 1261-9 & $(2.6 B V)(3.3 J K)$ \\
\hline BS8866 & $(3.4 V R)$ & NGC 288-A260 & $(2.8 B V)$ \\
\hline BS8878 & $(2.5 B V)$ & NGC $362-\mathrm{V} 2$ & $(3.4 J H)$ \\
\hline BS8905 & $(3.2 V R),(2.7 V I),\left(3.0 V L^{\prime}\right)$ & M3-33 & $(2.6 J H)$ \\
\hline HD 03008 & $(2.9 B V),(3.8 J H),(3.9 J K)$ & M3-46 & $(3.2 V K),(3.5 I K)$ \\
\hline HD 082590 & $(2.8 U V)$ & M3-53 & $(3.8 V K),(2.6 I K)$ \\
\hline HD 108577 & $(3.3 U V),(3.6 J H)$ & M3-68 & $(2.9 J K)$ \\
\hline HD 119516 & $(2.8 B V)$ & M3-72 & $(2.7 V K),(3.9 J K)$ \\
\hline HD 126778 & $(2.8 U V),(2.9 u b)$ & M3-428 & $(2.9 \mathrm{JH})$ \\
\hline HD 139641 & $(3.3 V K),(2.8$ by $)$ & M3-464 & $(2.9 V I)$ \\
\hline HD 141531 & $(2.8 V K),(3.7 J H),(2.6$ by $)$ & M3-496 & $(3.1 J K)$ \\
\hline HD 151937 & $(2.7 U V)$ & M3-525 & $(2.7 J H)$ \\
\hline HD 165195 & $(2.7 I K)$ & M3-586 & $(2.5 J H)$ \\
\hline HD 171496 & $(3.5 V K)$ & M3-627 & $(2.8 J H)$ \\
\hline HD 199191 & $(2.7 U V)$ & M3-659 & $(3.7 J K)$ \\
\hline HD 268518 & $(2.8 J K)$ & M3-675 & $(3.0 J H)$ \\
\hline HD 7424 & $(2.6 B V)$ & & \\
\hline SAO028774 & $(2.6 u b)$ & & \\
\hline SAO054175 & $(2.7 V I)$ & & \\
\hline SAO063927 & $(3.4 J K)$ & & \\
\hline SAO069416 & $(2.9 J H)$ & & \\
\hline
\end{tabular}

of the plain numerical inversion of the fits derived in the preceding sections, and the direct use of the photometric database of the stars of the sample. We have checked that the quotients between temperatures derived by applying the different calibrations are consistent within the limit of accuracy of the temperatures and photometry used in the present programme.

The derivation of colours in the range of low temperatures $\left(T_{\text {eff }} \leq 4500 \mathrm{~K}\right)$ is uncertain because the gradient $\mid \Delta T_{\text {eff }} / \Delta$ (colour) $\mid$ has in general small values (i.e. a minor variation in temperature implies a large variation in colour), and the size of temperature errorbars of stars in this range is large. The effect of this point is clearly illustrated by the case of $(R-I)$. This colour can be derived either from Eq. (7), or combining Eqs. (5) and (6). Discrepancies are found between both approaches.

In Figs. 18a-d, we show the comparison between intrinsic colours of Table 2 and the empirical calibration of von Braun et al. (1998) fixing $(V-K)$. Differences for $(B-V)$ and $(U-B)$ (colours which are more affected by blanketing effects) are stronger, while a better agreement is found for infrared colours $(V-I)$ and $(J-K)$. In Figs. 18c,d, we show also the comparison between intrinsic colours of Table 2 and the intrinsic colours of Bessell \& Brett (1988) for solar metallicity stars. A fairly good agreement is seen for $(J-K)$, however differences in $(V-I)$ are around $0.10 \mathrm{mag}$ for $(V-I) \gtrsim 3$.

The effects of gravity on broad band colours and effective temperatures are in general of the order of observational errors, remaining thus concealed when considering individual stars. However, the average relations derived in the above sections are accurate enough to permit us to address this question. For this purpose, we show in Fig. 19 the comparison between colour: $[\mathrm{Fe} / \mathrm{H}]: T_{\text {eff }}$ relations of giant stars derived in the above sections and those corresponding to main sequence stars (Paper III).

A noteworthy feature of the present relation $T_{\text {eff }}:[\mathrm{Fe} / \mathrm{H}]:(B-V)$ is that its global shape slightly differs from that corresponding to dwarf stars -classes V-VI- calibration (Fig. 19a), so that for a fixed $(B-V)$, giants' temperatures are higher than dwarfs' in the range $(B-V) \gtrsim 0.7$ and smaller in the range $(B-V) \lesssim 0.7\left(\Delta T_{\text {eff }} \approx 300 \mathrm{~K}\right.$ at $(B-V)=0.2$, $\Delta T_{\text {eff }} \approx 0 \mathrm{~K}$ at $(B-V)=0.7$ and $\Delta T_{\text {eff }} \approx-200 \mathrm{~K}$ at $(B-V)=1.2)$. The probable reason for this behaviour can be understood taking into account the variation of Paschen's continuum, and the TiO bands at $4954 \AA$ 
Table 5. Intrinsic broad band colours of giant stars in the system of Johnson for metallicities 0.2 , and 0 . Columns 2-11 (U-V), $(B-V),(V-R),(V-I),(R-I),(V-K),(J-K),(J-H),(I-K),(V-L)$. Column 12. Bolometric correction to $V$. The values in brackets are close to the range of validity of calibrations and therefore less reliable

\begin{tabular}{|c|c|c|c|c|c|c|c|c|c|c|c|}
\hline$T_{\text {eff }}(\mathrm{K})$ & $(U-V)$ & $(B-V)$ & $(V-R)$ & $(V-I)$ & $(R-I)$ & $(V-K)$ & $(J-K)$ & $(J-H)$ & $(I-K)$ & $(V-L)$ & $\mathrm{BC}(\mathrm{V})$ \\
\hline \multicolumn{12}{|c|}{$[\mathrm{Fe} / \mathrm{H}]=+0.2$} \\
\hline 3500 & - & $(1.930)$ & $(1.685)$ & $(2.800)$ & & - & $(1.250)$ & $(0.975)$ & $(1.955)$ & - & \\
\hline 3750 & - & $(1.705)$ & $(1.365)$ & $(2.210)$ & $(1.040)$ & $(4.125)$ & $(1.080)$ & $(0.855)$ & $(1.715)$ & $(4.230)$ & $(-1.425)$ \\
\hline 4000 & 3.315 & 1.515 & 1.155 & 1.865 & 0.825 & 3.450 & 0.935 & 0.745 & 1.505 & 3.560 & -0.957 \\
\hline 4250 & 2.885 & 1.345 & 1.000 & 1.625 & 0.695 & 2.995 & 0.815 & 0.660 & 1.325 & 3.100 & -0.687 \\
\hline 4500 & 2.475 & 1.190 & 0.875 & 1.435 & 0.600 & 2.640 & 0.715 & 0.585 & 1.170 & 2.735 & -0.495 \\
\hline 4750 & 2.090 & 1.055 & 0.770 & 1.275 & 0.520 & 2.355 & 0.625 & 0.515 & 1.035 & 2.435 & -0.351 \\
\hline 5000 & 1.720 & 0.935 & 0.680 & 1.135 & 0.460 & 2.115 & 0.550 & 0.450 & 0.915 & 2.170 & -0.259 \\
\hline 5250 & 1.320 & 0.825 & 0.605 & 1.015 & 0.405 & 1.890 & 0.480 & 0.400 & 0.805 & 1.940 & -0.186 \\
\hline 5500 & 0.995 & 0.725 & 0.540 & 0.910 & 0.360 & 1.690 & 0.420 & 0.350 & 0.705 & 1.735 & -0.131 \\
\hline 5750 & 0.800 & 0.625 & 0.485 & 0.815 & 0.320 & 1.505 & 0.365 & 0.300 & 0.620 & 1.550 & -0.089 \\
\hline 6000 & 0.650 & 0.550 & 0.430 & 0.725 & 0.285 & 1.335 & 0.315 & 0.265 & 0.540 & 1.380 & -0.057 \\
\hline 6250 & 0.530 & 0.475 & 0.385 & 0.645 & 0.250 & 1.175 & 0.270 & 0.225 & 0.465 & 1.220 & -0.032 \\
\hline 6500 & 0.425 & 0.410 & 0.345 & 0.575 & 0.225 & 1.030 & 0.235 & 0.190 & 0.400 & 1.075 & -0.014 \\
\hline 6750 & 0.400 & 0.350 & 0.305 & 0.505 & 0.195 & 0.895 & 0.195 & 0.160 & 0.340 & 0.950 & -0.001 \\
\hline 7000 & 0.380 & 0.295 & 0.270 & 0.440 & 0.175 & 0.770 & 0.160 & 0.135 & 0.280 & 0.815 & +0.007 \\
\hline 7250 & $(0.360)$ & 0.245 & 0.240 & 0.380 & 0.150 & 0.650 & 0.130 & 0.105 & 0.230 & 0.695 & +0.011 \\
\hline 7500 & $(0.340)$ & 0.200 & 0.210 & 0.325 & 0.135 & 0.540 & 0.100 & 0.080 & 0.180 & 0.585 & +0.013 \\
\hline 7750 & $(0.320)$ & $(0.160)$ & 0.185 & 0.275 & 0.115 & 0.440 & 0.070 & 0.060 & 0.135 & 0.480 & +0.011 \\
\hline 8000 & - & $(0.120)$ & $(0.160)$ & $(0.225)$ & $(0.100)$ & $(0.345)$ & $(0.050)$ & $(0.040)$ & $(0.090)$ & $(0.385)$ & $(+0.007)$ \\
\hline \multicolumn{12}{|c|}{$[\mathrm{Fe} / \mathrm{H}]=0.0$} \\
\hline 3500 & - & $(1.925)$ & $(1.660)$ & $(2.800)$ & & - & $(1.250)$ & $(0.980)$ & $(1.955)$ & - & - \\
\hline 3750 & - & $(1.695)$ & $(1.350)$ & $(2.210)$ & $(1.005)$ & $(4.095)$ & $(1.080)$ & $(0.860)$ & $(1.715)$ & $(4.230)$ & $(-1.411)$ \\
\hline 4000 & 3.240 & 1.495 & 1.145 & 1.865 & 0.815 & 3.435 & 0.935 & 0.755 & 1.505 & 3.560 & -0.947 \\
\hline 4250 & 2.790 & 1.320 & 0.990 & 1.625 & 0.690 & 2.980 & 0.815 & 0.670 & 1.325 & 3.100 & -0.680 \\
\hline 4500 & 2.365 & 1.165 & 0.865 & 1.435 & 0.595 & 2.630 & 0.715 & 0.590 & 1.170 & 2.735 & -0.492 \\
\hline 4750 & 1.955 & 1.030 & 0.760 & 1.275 & 0.520 & 2.345 & 0.625 & 0.520 & 1.035 & 2.435 & -0.351 \\
\hline 5000 & 1.565 & 0.905 & 0.680 & 1.135 & 0.460 & 2.105 & 0.550 & 0.460 & 0.915 & 2.170 & -0.260 \\
\hline 5250 & 1.160 & 0.790 & 0.600 & 1.015 & 0.405 & 1.885 & 0.480 & 0.405 & 0.805 & 1.940 & -0.189 \\
\hline 5500 & 0.910 & 0.690 & 0.535 & 0.910 & 0.360 & 1.685 & 0.420 & 0.355 & 0.705 & 1.735 & -0.135 \\
\hline 5750 & 0.730 & 0.605 & 0.480 & 0.815 & 0.320 & 1.500 & 0.365 & 0.310 & 0.620 & 1.550 & -0.094 \\
\hline 6000 & 0.590 & 0.530 & 0.425 & 0.725 & 0.285 & 1.335 & 0.315 & 0.270 & 0.540 & 1.380 & -0.063 \\
\hline 6250 & 0.475 & 0.460 & 0.380 & 0.645 & 0.255 & 1.180 & 0.270 & 0.235 & 0.465 & 1.220 & -0.040 \\
\hline 6500 & 0.375 & 0.395 & 0.340 & 0.575 & 0.225 & 1.035 & 0.235 & 0.200 & 0.400 & 1.075 & -0.023 \\
\hline 6750 & 0.320 & 0.335 & 0.300 & 0.505 & 0.200 & 0.900 & 0.195 & 0.170 & 0.340 & 0.950 & -0.012 \\
\hline 7000 & 0.300 & 0.285 & 0.265 & 0.440 & 0.175 & 0.775 & 0.160 & 0.140 & 0.280 & 0.815 & -0.005 \\
\hline 7250 & $(0.280)$ & 0.235 & 0.235 & 0.380 & 0.155 & 0.660 & 0.130 & 0.110 & 0.230 & 0.695 & -0.001 \\
\hline 7500 & $(0.260)$ & 0.190 & 0.205 & 0.325 & 0.135 & 0.550 & 0.100 & 0.090 & 0.180 & 0.585 & -0.001 \\
\hline 7750 & $(0.250)$ & $(0.145)$ & 0.180 & 0.275 & 0.120 & 0.445 & 0.070 & 0.065 & 0.135 & 0.480 & -0.003 \\
\hline 8000 & - & $(0.110)$ & $(0.155)$ & $(0.225)$ & $(0.100)$ & $(0.350)$ & $(0.050)$ & $(0.045)$ & $(0.090)$ & $(0.385)$ & $(-0.008)$ \\
\hline
\end{tabular}

and $5167 \AA$ with gravity and temperature in the ranges considered. For $8000 \mathrm{~K} \gtrsim T_{\text {eff }} \gtrsim T_{\odot}$ the shape of Paschen's continuum is dominated by the bound-free absorption of atomic hydrogen and the $\mathrm{H}^{-}$ion. The wavelength dependence of the former is stronger, and its contribution becomes increasingly more important towards higher temperatures. Furthermore, the $\mathrm{H}$ bound-free opacity does not depend on the electron pressure, whereas the $\mathrm{H}^{-}$bound-free absorption does. For a given $T_{\text {eff }}$, the lower the atmospheric gravity the smaller the electron pressure, making the $\mathrm{H}^{-}$bound-free contribution to decrease, and the continuum slope to become steeper. For the range $T_{\odot} \gtrsim T_{\text {eff }} \gtrsim 4000 \mathrm{~K}$, the true continuum is not very sensitive to gravity variations, but the strong $\mathrm{TiO}$ bands integrated within the $V$ filter become more prominent with larger gravities (increasing the value of $V$ magnitude). In conclusion, the combination of both mechanisms results in the observed effect: for a fixed temperature, giants have bluer $(B-V)$ colours in the range $T_{\text {eff }} \gtrsim 5500 \mathrm{~K}$, and redder ones in the $T_{\text {eff }} \lesssim$ $5500 \mathrm{~K}\left(\Delta(B-V) \approx 0.05 \mathrm{mag}\right.$ at $T_{\text {eff }}=7000 \mathrm{~K}$, $\Delta(B-V) \approx 0.0 \mathrm{mag}$ at $T_{\mathrm{eff}}=5500 \mathrm{~K}$, and $\Delta(B-V) \approx-0.15 \mathrm{mag}$ at $\left.T_{\mathrm{eff}}=4000 \mathrm{~K}\right)$.
The relation $T_{\text {eff }}:[\mathrm{Fe} / \mathrm{H}]:(R-I)$ for giants stars has no significant differences with that of dwarfs (Fig. 19b). Hence $(R-I)$ is a temperature indicator free of surface gravity effects.

The effect of gravity on the relation $T_{\text {eff }}:[\mathrm{Fe} / \mathrm{H}]:(V-I)$ is also appreciable as in the case of $(B-V)$ (Fig. 19c). In the whole range of temperatures considered, $(V-I)$ colours of dwarf stars are bluer than those of giant stars of the same temperature $\left(\Delta(V-I) \approx 0.1 \mathrm{mag}\right.$ at $T_{\text {eff }}=$ $7000 \mathrm{~K}, \Delta(V-I) \approx 0.04 \mathrm{mag}$ at $T_{\text {eff }}=5000 \mathrm{~K}$, and $\Delta(V-I) \approx 0.15 \mathrm{mag}$ at $\left.T_{\text {eff }}=4000 \mathrm{~K}\right)$. Conversely, temperatures of dwarfs are greater at fixed colour $\left(\Delta T_{\text {eff }} \approx\right.$ $280 \mathrm{~K}$ at $(V-I)=0.5, \Delta T_{\mathrm{eff}} \approx 100 \mathrm{~K}$ at $(V-I)=1$ and $\Delta T_{\text {eff }} \approx 120 \mathrm{~K}$ at $\left.(V-I)=2\right)$. The effect is again, probably related to the behaviour of opacity with gravity in $V$ and $I$ bands. The effect of gravity, although not shown in Fig. 19, is also appreciable in the case of $(V-R),(J-H)$ and $(J-K)$.

Finally, the present relation $T_{\text {eff }}:[\mathrm{Fe} / \mathrm{H}]=:(V-K)$ for giant stars differs slightly from that of dwarfs (Fig. 19d): Over $6000 \mathrm{~K}$ dwarfs' temperatures are slightly higher than giants' $(\sim 50 \mathrm{~K}$ at $(V-K) \approx 0.2)$, conversely $(V-K)$ is redder for dwarfs $(\sim 0.03 \mathrm{mag})$. Under $4000 \mathrm{~K}$ dwarfs' 
Table 6. Intrinsic broad band colours of giant stars in the system of Johnson for metallicities $-1,-2$ and -3 . Columns $2-11$ $(U-V),(B-V),(V-R),(V-I),(R-I),(V-K),(J-K),(J-H),(I-K),(V-L)$. Column 12. Bolometric correction to $V$. The values in brackets are less reliable

\begin{tabular}{|c|c|c|c|c|c|c|c|c|c|c|c|}
\hline$T_{\text {eff }}(\mathrm{K})$ & $(U-V)$ & $(B-V)$ & $(V-R)$ & $(V-I)$ & $(R-I)$ & $(V-K)$ & $(J-K)$ & $(J-H)$ & $(I-K)$ & $(V-L)$ & $\mathrm{BC}(\mathrm{V})$ \\
\hline \multicolumn{12}{|c|}{$[\mathrm{Fe} / \mathrm{H}]=-1.0$} \\
\hline 3500 & $=$ & $(1.925)$ & $(1.570)$ & - & & - & & $=$ & - & $=$ & - \\
\hline 3750 & $(3.505)$ & $(1.665)$ & $(1.300)$ & $(2.210)$ & $(0.925)$ & $(3.985)$ & $(1.080)$ & $(0.875)$ & - & - & $(-1.350)$ \\
\hline 4000 & 2.935 & 1.440 & 1.105 & 1.865 & 0.775 & 3.365 & 0.935 & 0.770 & 1.505 & — & -0.905 \\
\hline 4250 & 2.380 & 1.240 & 0.960 & 1.625 & 0.670 & 2.930 & 0.815 & 0.685 & 1.325 & - & -0.657 \\
\hline 4500 & 1.830 & 1.065 & 0.840 & 1.435 & 0.590 & 2.590 & 0.715 & 0.605 & 1.170 & - & -0.486 \\
\hline 4750 & 1.355 & 0.905 & 0.740 & 1.275 & 0.520 & 2.315 & 0.625 & 0.535 & 1.035 & - & -0.358 \\
\hline 5000 & 1.035 & 0.775 & 0.660 & 1.135 & 0.465 & 2.085 & 0.550 & 0.475 & 0.915 & - & -0.273 \\
\hline 5250 & 0.800 & 0.695 & 0.585 & 1.015 & 0.415 & 1.875 & 0.480 & 0.420 & 0.805 & - & -0.209 \\
\hline 5500 & 0.625 & 0.605 & 0.520 & 0.910 & 0.375 & 1.680 & 0.420 & 0.375 & 0.705 & - & -0.162 \\
\hline 5750 & 0.485 & 0.530 & 0.465 & 0.815 & 0.335 & 1.505 & 0.365 & 0.330 & 0.620 & - & -0.128 \\
\hline 6000 & 0.370 & 0.460 & $(0.415)$ & $(0.725)$ & 0.305 & 1.345 & 0.315 & 0.290 & 0.540 & - & -0.104 \\
\hline 6250 & $(0.270)$ & 0.400 & - & - & 0.275 & 1.195 & 0.270 & 0.250 & $(0.465)$ & - & -0.087 \\
\hline 6500 & - & $(0.340)$ & - & - & $(0.245)$ & 1.060 & 0.235 & 0.220 & - & - & -0.076 \\
\hline 6750 & - & - & - & - & - & $(0.930)$ & — & - & - & - & $(-0.070)$ \\
\hline \multicolumn{12}{|c|}{$[\mathrm{Fe} / \mathrm{H}]=-2.0$} \\
\hline 3750 & - & - & - & - & - & - & - & - & - & $\overline{-}$ & $(-1.306)$ \\
\hline 4000 & 2.830 & $(1.450)$ & $(1.090)$ & 1.865 & $(0.765)$ & $(3.340)$ & $(0.935)$ & $(0.765)$ & $(1.505)$ & - & -0.881 \\
\hline 4250 & 2.130 & 1.220 & 0.950 & 1.625 & 0.670 & 2.915 & 0.815 & 0.675 & 1.325 & - & -0.651 \\
\hline 4500 & 1.430 & 1.015 & 0.835 & 1.425 & 0.595 & 2.580 & 0.715 & 0.600 & 1.170 & - & -0.497 \\
\hline 4750 & 1.085 & 0.835 & 0.735 & 1.275 & 0.535 & 2.310 & 0.625 & 0.530 & 1.035 & - & -0.379 \\
\hline 5000 & 0.820 & 0.710 & 0.655 & 1.135 & 0.480 & 2.085 & 0.550 & 0.470 & 0.915 & - & -0.296 \\
\hline 5250 & 0.625 & 0.645 & 0.580 & 1.015 & 0.435 & 1.885 & 0.480 & 0.415 & 0.805 & — & -0.240 \\
\hline 5500 & 0.475 & 0.565 & 0.520 & 0.910 & 0.395 & 1.700 & 0.420 & 0.370 & 0.705 & - & -0.200 \\
\hline 5750 & $(0.350)$ & 0.495 & $(0.465)$ & $(0.815)$ & 0.360 & 1.535 & 0.365 & 0.325 & $(0.620)$ & - & -0.173 \\
\hline 6000 & - & 0.435 & - & - & - & 1.380 & 0.315 & 0.285 & - & - & -0.155 \\
\hline 6250 & - & 0.375 & - & - & - & 1.235 & 0.270 & $(0.250)$ & - & - & -0.144 \\
\hline 6500 & - & $(0.325)$ & - & - & - & $(1.105)$ & $(0.235)$ & $(0.215)$ & - & - & $(-0.139)$ \\
\hline \multicolumn{12}{|c|}{$[\mathrm{Fe} / \mathrm{H}]=-3.0$} \\
\hline 4000 & - & - & - & - & - & - & - & - & - & - & $(-0.874)$ \\
\hline 4250 & - & - & - & 1.625 & - & $(2.930)$ & $(0.815)$ & 0.645 & $(1.325)$ & - & -0.662 \\
\hline 4500 & $(1.320)$ & $(1.050)$ & 0.845 & 1.435 & 0.615 & 2.600 & 0.715 & 0.570 & 1.170 & - & -0.525 \\
\hline 4750 & 0.975 & 0.815 & 0.750 & 1.275 & 0.560 & 2.330 & 0.625 & 0.500 & 1.035 & - & -0.415 \\
\hline 5000 & 0.735 & 0.710 & 0.670 & 1.135 & 0.505 & 2.110 & 0.550 & 0.445 & 0.915 & - & -0.330 \\
\hline 5250 & 0.560 & 0.630 & 0.595 & 1.015 & 0.465 & 1.920 & 0.480 & 0.390 & 0.805 & - & -0.281 \\
\hline 5500 & - & 0.555 & $(0.535)$ & $(0.910)$ & 0.425 & 1.740 & 0.420 & $(0.340)$ & - & - & $(-0.249)$ \\
\hline 5750 & - & $(0.495)$ & - & - & $(0.390)$ & - & - & $(0.300)$ & - & - & - \\
\hline
\end{tabular}

temperatures are smaller $(\sim 100 \mathrm{~K}$ at $(V-K) \approx 4)$, conversely $(V-K)$ is bluer for dwarfs $(\sim 0.1$ mag at $3900 \mathrm{~K})$. In the range $6000 \mathrm{~K} \gtrsim T_{\text {eff }} \gtrsim 4000 \mathrm{~K}$ dwarf and giant temperatures and colours are indistinguishable. The small size of the effect confirms that $(V-K)$ is a temperature indicator which has only marginal dependence on stellar surface gravity.

\section{Summary}

We provide a comprehensive calibration of the scale of effective temperature of giant stars (F0-K5) against different colours, which takes into account the effect of metallicity. Three features of the present calibrations must be pointed out: (a) They are based on a large sample of field and globular cluster giants representative of the galactic populations, which have accurate photometric data; (b) The effective temperatures considered in the calibrations have been obtained by using the IRFM scaled to the direct temperatures derived from interferometric techniques; (c) The $[\mathrm{Fe} / \mathrm{H}]$ values adopted in the analysis are ultimately linked to the spectroscopic determinations compiled by Cayrel de Strobel et al. (1997).

As a consequence, these calibrations provide a substantial extension of the previous empirical works devoted to giant stars both in temperature and metallicity ranges. Furthermore, the following points must be stressed after the analysis of the results:

(I) The comparison of the present temperature scale of giants with that of main sequence stars obtained previously with the same procedure (Paper III) shows that in general, gravity is a non negligible parameter in the calibration of temperatures against colours. Indeed, its effect is considerable for $(B-V),(V-R),(V-I),(J-K)$ and $(J-H)$; however, $(R-I)$ and $(V-K)$ have revealed themselves as temperature indicators with negligible dependence on gravity.

(II) For giant stars, in the range of temperatures studied, $(V-I),(I-K),(J-K)$ and to a lesser extent $(V-$ $K)$ are temperature indicators free of blanketing effects (i.e. non-dependent on metallicity). For this reason, their use is preferable as temperature indicators for stars of unknown metallicity. However, the effect of metallicity cannot be neglected for $(U-V),(B-V),(V-R),(R-I)$, $(J-H),(u-b)$ and $(b-y)$.

(III) Concerning the comparison of the present calibrations with previous works, we have to make the following distinction. On the one hand, theoretical calibrations are in general very different both from each other and from empirical relations derived in this work. 
Table 7. Intrinsic Strömgren colours of giant stars

\begin{tabular}{c|ccccc|cc|cc|cc}
\hline \multicolumn{3}{c}{$[\mathrm{Fe} / \mathrm{H}]$} & $=0.20$ & \multicolumn{2}{c}{$[\mathrm{Fe} / \mathrm{H}]$} & $=0.00$ & \multicolumn{1}{c}{$[\mathrm{Fe} / \mathrm{H}]$} & $=-1.00$ & \multicolumn{2}{c}{$[\mathrm{Fe} / \mathrm{H}]=-2.00$} & \multicolumn{2}{c}{$[\mathrm{Fe} / \mathrm{H}]=-2.50$} \\
\hline$T_{\text {eff }}$ & $(u-b)$ & $(b-y)$ & $(u-b)$ & $(b-y)$ & $(u-b)$ & $(b-y)$ & $(u-b)$ & $(b-y)$ & $(u-b)$ & $(b-y)$ \\
\hline 8000 & - & $(0.070)$ & $(1.56)$ & $(0.065)$ & - & - & - & - & - & - \\
7750 & - & 0.100 & 1.54 & 0.095 & - & - & - & - & - & - \\
7500 & - & 0.130 & 1.50 & 0.125 & - & - & - & - & - & - \\
7250 & - & 0.160 & 1.48 & 0.155 & - & - & - & - & - & - \\
7000 & - & 0.195 & 1.46 & 0.185 & - & - & - & - & - & - \\
6750 & - & 0.230 & 1.44 & 0.225 & - & - & - & - & - & - \\
6500 & - & 0.270 & 1.42 & 0.260 & - & $(0.240)$ & - & - & - & - \\
6250 & - & 0.310 & 1.43 & 0.300 & - & $(0.275)$ & - & $(0.280)$ & - & - \\
6000 & - & 0.355 & 1.47 & 0.345 & - & 0.315 & - & $(0.315)$ & - & - \\
5750 & $1.605)$ & 0.400 & 1.54 & 0.390 & - & 0.360 & - & 0.355 & - & - \\
5500 & 1.825 & 0.455 & 1.72 & 0.440 & - & 0.405 & - & 0.400 & - & 0.405 \\
5250 & 2.070 & 0.510 & 1.965 & 0.500 & $(1.54)$ & 0.460 & - & 0.450 & - & 0.450 \\
5000 & 2.345 & 0.580 & 2.24 & 0.560 & 1.815 & 0.515 & $(1.555)$ & 0.500 & $(1.47)$ & 0.500 \\
4750 & 2.650 & 0.645 & 2.545 & 0.630 & 2.125 & 0.575 & 1.870 & 0.560 & 1.79 & 0.580 \\
4500 & 3.000 & 0.725 & 2.895 & 0.710 & 2.475 & 0.665 & 2.225 & 0.665 & 2.155 & 0.690 \\
4250 & 3.405 & 0.820 & 3.295 & 0.810 & 2.875 & 0.770 & 2.630 & 0.790 & 2.575 & $(0.835)$ \\
4000 & - & 0.945 & $(3.765)$ & 0.935 & 3.340 & $(0.910)$ & 3.105 & $(0.965)$ & $(3.06)$ & - \\
3750 & - & - & - & $(1.105)$ & $(3.890)$ & - & $(3.665)$ & - & - & - \\
\hline \hline
\end{tabular}

The trend and the size of the observed discrepancies seem too large to be ascribed only to accidental errors on photometry and uncertainties affecting the effective temperature determinations. Therefore they suggest the persistence of essential problems in the theory of stellar atmospheres (e.g. opacities in bands $U$ and $B$, convection treatment) and/or the synthesis of colours (e.g. difficulties on the adequate reconstruction of Johnson $U B V$ system). On the other hand, the level of agreement found between (semi-)empirical calibrations based on independent approaches and the present work is fairly good, although there remain uncertainties in the zero point of the scale, and systematic differences under $4000 \mathrm{~K}$ and over $5500 \mathrm{~K}$.

In summary, this work demonstrates the necessity of considering the effect of metallicity and gravity on the relations which link effective temperatures with intrinsic colours and bolometric corrections of giants stars. Therefore, we must conclude by emphasizing that differences found between the present empirical calibration and other (semi)-empirical and theoretical ones might have relevant consequences on population synthesis and on the transformation of isochrones from the theoretical HR diagram into observed colour-magnitude diagrams. The most obvious effect regarding the latter, being the variation of the shape and location of the red giant branch, which has in turn influence on the interpretation of the ages (when derived from the subgiant branch colour extension), reddenings and metal content of globular clusters, and even on the choice of the free parameter $\alpha$ in the theory of convection (i.e. the ratio of the mixing length to the pressure scale height). Furthermore, the implications of the observed discrepancies should also be taken into account when applying calibrations to the determination of tem- peratures of individual stars from photometric colours, and to the analysis of colours synthesized from model atmospheres.

Acknowledgements. We thank Dr. Carlos Allende and Dr. Maurizio Salaris for many helpful discussions on different points of this work. We are grateful to the referee, Dr. LynasGray, for many helpful comments and suggestions which have certainly improved the final version of this paper. We have made use in this research of the SIMBAD database, operated at CDS, Strasbourg, France.

\section{References}

Alonso A., Arribas S., Martínez-Roger C., 1994, A\&A 282, 684 Alonso A., Arribas S., Martínez-Roger C., 1996, A\&A 313, 873 (Paper III)

Alonso A., Arribas S., Martínez-Roger C., 1998, A\&AS 131, 209 (Paper I)

Alonso A., Arribas S., Martínez-Roger C., 1999, A\&AS 139, 335 (Paper II)

Anthony-Twarog B.J., Twarog B.A., 1994, AJ 107, 1577

Bell R.A., 1992, MNRAS 257, 423

Bell R.A., Gustaffson B., 1989, MNRAS 236, 653 (BG89)

Bessell M.S., 1979, PASP 91, 589

Bessell M.S., Brett J., 1988, PASP 100, 1134

Bessell M.S., Castelli F., Plez B., 1998, A\&A 333, 231 (BCP98)

Blackwell D.E., Petford A.D., Arribas S., Haddock D.J., Selby M.J., 1990, A\&A 232, 396

Blackwell D.E., Lynas-Gray A.E., 1998, A\&AS 129, 505 (BL98)

Böhm-Vitense E., 1981, ARA\&A 19, 295

Bonifacio P., Molaro P., 1997, MNRAS 285, 847

Buser R., Kurucz R.L., 1992, A\&A 264, 557 (BK92)

Cassisi S., Castellani V., Degl'Innocenti S., Salaris M., Weiss A., 1999, A\&AS 134, 103 
Cayrel de Strobel G., Soubiran C., Friel E.D., et al., 1997, A\&AS 124, 299

Davis Philip A.G., 1996, Balt. A. 5, 425

Di Benedetto G.P., 1993, A\&A 270, 315

Di Benedetto G.P., 1998, A\&A 339, 858 (DB98)

Fernie J.D., 1983, PASP 95, 782

Flower P.J., 1996, ApJ 469, 365

Johnson H.L., 1966, ARA\&A 4, 193 (J66)

Montegriffo P., Ferraro F.R., Origlia L., Fusi Pecci F., 1998, MNRAS 297, 872 (M98)

Ridgway S.T., Joyce R.R., White N.M., Wing R.F., 1980, ApJ
235,126

Ryan S.G., Beers T.C., Deliyannis C., Thorburn J.A., 1996, ApJ 458, 543

Sneden C., Kraft R.P., Prosser C.F., Langer G.E., 1992, AJ 102,2001

Spite F., Spite M., Hill V., 1998, Space Sci. Rev. 84, 155

Vazdekis A., Casuso E., Peletier R.F., Beckman J.E., 1996, ApJS 106, 307

von Braun K., Chiboucas K., Minske J.K., Salgado J.F., 1998, PASP 110,810 